\title{
Nonlinear Resonant Responses, Mode Interactions, and Multitime Periodic and Chaotic Oscillations of a Cantilevered Pipe Conveying Pulsating Fluid under External Harmonic Force
}

\author{
Y. F. Zhang, ${ }^{1}$ T. Liu $\mathbb{D}^{2}{ }^{2}$ and W. Zhang $\mathbb{D}^{2}$ \\ ${ }^{1}$ School of Aerospace Engineering, Shenyang Aerospace University, Shenyang, Liaoning 110136, China \\ ${ }^{2}$ Beijing Key Laboratory of Nonlinear Vibrations and Strength of Mechanical Structures, College of Mechanical Engineering, \\ Beijing University of Technology, Beijing 100124, China
}

Correspondence should be addressed to T. Liu; liu_tao@yahoo.com and W.Zhang; sandyzhang0@yahoo.com

Received 2 June 2020; Accepted 22 July 2020; Published 28 August 2020

Guest Editor: Yongjian Liu

Copyright $(2020$ Y. F. Zhang et al. This is an open access article distributed under the Creative Commons Attribution License, which permits unrestricted use, distribution, and reproduction in any medium, provided the original work is properly cited.

\begin{abstract}
The nonlinear resonant responses, mode interactions, and multitime periodic and chaotic oscillations of the cantilevered pipe conveying pulsating fluid are studied under the harmonic external force in this research. According to the nonlinear dynamic model of the cantilevered beam derived using Hamilton's principle under the uniformly distributed external harmonic excitation, we combine Galerkin technique and the method of multiple scales together to obtain the average equation of the cantilevered pipe conveying pulsating fluid under 1:3 internal resonance and principal parametric resonance. Based on the average equation in the polar form, several amplitude-frequency response curves are obtained corresponding to the certain parameters. It is found that there exist the hardening-spring type behaviors and jumping phenomena in the cantilevered pipe conveying pulsating fluid. The nonlinear oscillations of the cantilevered pipe conveying pulsating fluid can be excited more easily with the increase of the flow velocity, external excitation, and coupling degree of two order modes. Numerical simulations are performed to study the chaos of the cantilevered pipe conveying pulsating fluid with the external harmonic excitation. The simulation results exhibit the existence of the period, multiperiod, and chaotic responses with the variations of the fluid velocity or excitation. It is found that, in the cantilevered pipe conveying pulsating fluid, there are the multitime nonlinear vibrations around the left-mode and the right-mode positions, respectively. We also observe that there exist alternately the periodic and chaotic vibrations of the cantilevered pipe conveying pulsating fluid in the certain range.
\end{abstract}

\section{Introduction}

Pipes conveying fluid are widely utilized in many engineering fields, such as aeronautic, astronautic, and mechanical engineering systems. It is extremely important for us to ensure the efficient utilization and safe operation of the pipe conveying fluid system, and its stable and safe operations are closely related to all aspects of the personal life and industrial production. However, the applications of the pipes conveying pulsating fluid are particularly challenging because they undergo the large deformations and significant stresses. The large deformations often lead to the nonlinear vibrations of the pipes conveying pulsating fluid. One of the main reasons for the nonlinear vibrations of the pipes conveying pulsating fluid is the time-varying flow speed and external harmonic excitation. Pulsating flow due to the pump operation can cause a parametric excitation loading in the pipes conveying fluid. The nonlinear oscillations of the pipes conveying pulsating fluid will lead to the structure damages. As we all know, there are three typical types of nonlinear oscillations in the structures and systems, namely, the periodic, quasi-periodic, and chaotic oscillations. In fact, the chaotic oscillations of the pipes conveying pulsating fluid are dangerous because the amplitudes of the chaotic oscillations are larger than those of the periodic oscillations, which have been the object of increasing attention in engineering applications. However, there is less research on the nonlinear oscillations of the cantilevered pipe conveying 
pulsating fluid with $1: 3$ internal resonance when the fluid is transported at a critical speed through the pipe. Therefore, it is of great significance for us to study the nonlinear oscillations of the cantilevered pipe conveying pulsating fluid under the case of $1: 3$ internal resonance.

The pipe conveying fluid mainly consists of three important elements: pipeline, fluid, and external environment. It is necessary to establish a mathematical model for obtaining a reasonable description of the pipes conveying fluid. The beam model is usually used for analysis of the vibration when the pipe diameter is much smaller than the length. The nonlinear dynamic study of the pipe conveying fluid system began in 1980s. Researches for the pipes conveying pulsating fluid have become a hot field of engineering and science [1-4]. Holmes and Marsden $[5,6]$ established the first dynamic model of motion for the pipe conveying fluid through considering the nonlinear factors. They studied the bifurcation phenomena caused by the velocity and the axial force and summed up the bifurcation motion characteristics of the pipe conveying fluid system. According to Hamilton's principle and Euler-Bernoulli beam theory, Huo and Wang [7] derived the differential governing equation of motion for a vertical cantilevered pipe conveying fluid when the pipe exhibits the deploying or retracting motions and discussed the influence of the deploying or retracting speed, mass ratio, and fluid velocity on the dynamic responses and stability. Based on the modified strain gradient theory in conjunction with EulerBernoulli beam model, Hosseini and Bahaadini [8] studied the size-dependent stability of the cantilever micropipes conveying fluid and examined the influences of the geometric parameters on the natural frequencies and the flutter critical speeds. Askarian et al. [9] researched the dynamic stability of a vertical clamped-free pipe conveying pulsatile flow by using Euler-Bernoulli beam theory.

In addition, some scholars also provided several different mathematical models to investigate the vibrations of the pipes conveying fluid. According to the nonlinear Novozhilov shell theory for the isotropic materials, Tubaldi et al. [10] established a fully coupled fluid-structure interaction model and studied the nonlinear vibrations of the circular cylindrical shells conveying pulsatile flow with the flexible boundary conditions subjected to the pulsatile pressure. Bai et al. [11] simulated the varying density fluid of the vertical cantilevered pipe conveying fluid by a new mathematical model.

After the establishment of the rational mathematical model, the further researches on the vibrations of the pipes conveying fluid are mainly focused on three significant aspects. Firstly, the vibration characteristics and instability conditions of the pipes conveying fluid are studied, which have different boundary conditions, material properties, and functions. Secondly, the problems of the nonlinear dynamics are studied to understand the nonlinear vibration characteristics of the pipes conveying fluid because the pipes can be regarded as the complex nonlinear dynamical systems. Thirdly, the transfer mechanism of the energy between two modes and the internal resonance are studied to avoid the chaotic vibrations of the pipes conveying fluid.
More and more researches about model and analysis have been published with the continuous development of modern computing and analytical techniques. Bajaj et al. [12] researched the nonlinear vibration responses of the pipes conveying fluid and analyzed the influences of flow rate, mass ratio, and pressure on the nonlinear vibrations and found that Hopf bifurcation occurs under the certain conditions. Sri Namchchivaya and Tien $[13,14]$ examined the nonlinear vibrations of the supported pipes conveying pulsating fluid and found that the trivial solution of the averaged equation loses its stability through the simple or double zero bifurcations in the vicinity of the subharmonic resonance. Based on a semianalytical approach, Sarkar and Paidoussis [15] obtained the proper orthogonal modes to describe the nonlinear oscillations of a cantilevered pipe conveying fluid and to explore the nonlinear dynamics of the pipe by means of the low-dimensional model. McDonald and Sri Namachchivaya [16] studied the local bifurcation behaviors of parametrically excited simply supported pipes conveying fluid and the stability of solution where the energy transfer may happen from the high-frequency to low-frequency vibration modes.

Yoshizawa et al. [17] established the dynamic model of the pipes conveying fluid under the fixed-hinge boundary conditions and studied the vibration responses under the effect of pulse flow. For the pipe conveying fluid system, Hou and Zeng [18] obtained numerical solutions of the transverse vibration equation through using the finite element method. Setoodeh and Afrahim [19] investigated the nonlinear vibrations of the functionally graded materials micropipes conveying fluid. An explicit expression of the nonlinear fundamental frequency was given by using the homotopy analysis method. Kheiri and Païdoussis [20] derived the equation of motion for a typical flexible pipe conveying fluid by using the generalized Hamilton's principle. Gan et al. [21] simplified the equation of motion to the random variable and researched the vibration characteristics of the pipes conveying fluid clamped at both ends. Zhang et al. [22] established three-dimensional nonlinear equations of motion for the pipes conveying fluid under the general boundary conditions. The natural frequencies of the pipes under different boundary conditions were calculated and the nonlinear dynamic characteristics were analyzed. Wang and Liu [23] studied the transverse vibration and stability of the functionally graded material pipe conveying fluid by utilizing the symplectic method.

Based on the fluid-structure interaction, Liang et al. [24] studied the free vibration of the pipes conveying fluid through using the linear and nonlinear complex mode approach. Liang et al. [25] gave the analysis of the nonlinear free vibration for the spinning viscoelastic pipes conveying fluid. Liang et al. [26] studied the transverse free vibration of the spinning pipes conveying fluid and found that the qualitative stability of the pipes mainly relies on the fluidstructure interaction and mass ratio. Liang et al. [27] established a dynamic model of simply supported spinning pipes conveying fluid with the axial deployment and studied the transverse free vibration involving the time-dependent parameters. Liang et al. [28] researched the coupled 
flexural-torsional vibrations of the pipes conveying fluid spinning on an eccentric axis. Recently, Liang et al. [29] investigated the parametric vibrations of the pipes conveying fluid by the nonlinear normal modes and numerical iterative approach.

Several papers researched the nonlinear dynamic characteristics of the pipes conveying fluid. $\mathrm{Li}$ and Paidoussis [30] studied the nonlinear oscillations of a standing cantilevered pipe conveying fluid and showed that the chaotic oscillations of the cantilevered pipe exist when the gravity parameter was sufficiently perturbed off the doubly degenerate point. Based on the theoretical and experimental methods, Semler and Paidoussis [31, 32] analyzed the nonlinear oscillations of the cantilevered pipe conveying fluid with a sinusoidally perturbed flow velocity and studied the nonlinear dynamic responses of the cantilevered pipe conveying fluid with a small mass attached at the free end. They found that there exist the jumping phenomena and quasi-periodic and chaotic oscillations. Jin and Zou [33] studied the stability and nonlinear dynamics of the cantilevered pipe conveying fluid with the motion limiting constraints and a linear spring support and analyzed the local behaviors in the neighborhood of a double degenerate point. Ghayesh et al. [34] researched the nonlinear dynamic characteristics of the pipes conveying fluid through considering the lateral and longitudinal displacements and found that the cantilevered pipe will generate the flutter through Hopf bifurcation at the critical velocity. Wang et al. [35] studied the nonlinear oscillations of the pipes conveying fluid under the loose constraint boundary conditions. Askarian et al. [36] investigated the nonlinear oscillations of an extensible cantilevered pipe conveying pulsating flow with a nozzle attached to the end of the pipe.

For high-dimensional nonlinear dynamical systems, due to the existence of the modal interactions, there exist the relationships among several types of internal resonances, which can lead to different nonlinear oscillations [37-40]. Panda and Kar [41] established the nonlinear dynamic model of the pipes conveying fluid under the hinged boundaries at both ends and studied the nonlinear dynamic characteristics of the pipes. Ni et al. [42] simplified the pipes conveying fluid to the constant coefficients gyroscopic system and studied the nonlinear oscillations of the pipes on the nonlinear elastic foundation under $3: 1$ internal resonance. Zhang and Chen [43] used the multiple-scale method to determine the steady-state solutions of a pipe conveying fluid under 2:1 internal resonance. Mao et al. [44] investigated the forced oscillations of the pipe conveying fluid with 3:1 internal resonance around the bending configuration. Zhang et al. [45] established the nonlinear dynamic model of the cantilevered pipe conveying pulsating fluid under the external harmonic excitation and analyzed the multipulse orbits and chaotic oscillations with $1: 2$ internal resonance through utilizing the energy phase method.
Recently, Ding et al. [46] investigated the nonlinear vibration isolation of the pipes conveying fluid through using the quasi-zero stiffness characteristics.

To avoid the damage of the pipes conveying pulsating fluid caused by chaotic oscillations, we study the nonlinear oscillations of the pipes conveying pulsating fluid under $1: 3$ internal resonance. Based on the nonlinear dynamic model of the cantilevered beam, the nonlinear dynamic equations of motion for the cantilevered pipe conveying pulsating fluid under the uniformly distributed harmonic excitation and average equations are obtained through using the combination of the multiple-scale method and Galerkin technique under 1:3 internal resonance and principal parametric resonance. We analyze the nonlinear resonant responses and the mode interactions of the cantilevered pipe conveying pulsating fluid. Moreover, numerical simulations are performed to study the multitime periodic and chaotic oscillations of the cantilevered pipe conveying pulsating fluid under the external harmonic excitation. It is found that, in the cantilevered pipe conveying pulsating fluid, there are the multitime nonlinear and chaotic oscillations around the leftmode and the right-mode positions, respectively.

\section{Equation of Motion and Perturbing Analysis}

We consider a cantilevered pipe conveying pulsating fluid, where $A$ is the internal cross-sectional area, $L$ is the length of a tubular cantilevered beam, $m$ is the mass per unit length of the pipe, $E I$ is the flexural rigidity, $M$ is the conveying fluid mass per unit length with an axial velocity $U$ which may vary with respect to time, and $f$ is an external harmonic force, as shown in Figure 1. It is assumed that initially vertical position along the $X$-axis is located in the direction of gravity and the nonlinear vibrations occur in the $(X, Y)$ plane for the cantilevered pipe conveying pulsating fluid.

The basic assumptions of the cantilevered pipe and the fluid are made as follows:

(i) The fluid is incompressible.

(ii) The diameter of the pipe is small compared to its length. Therefore, the pipe behaves like an EulerBernoulli beam.

(iii) The vibration of the pipe is planar, and the deflections of the pipe are large.

(iv) The rotatory inertia and shear deformation are neglected.

(v) The pipe centerline has inextensible property in the case of a cantilevered pipe.

Based on researches given by Semler et al. [3], Païdoussis [4], and Zhang et al. [45], the nonlinear partial differential governing equation of motion for the cantilevered pipe conveying pulsating fluid is derived through utilizing Hamilton's principle under the external harmonic excitation: 


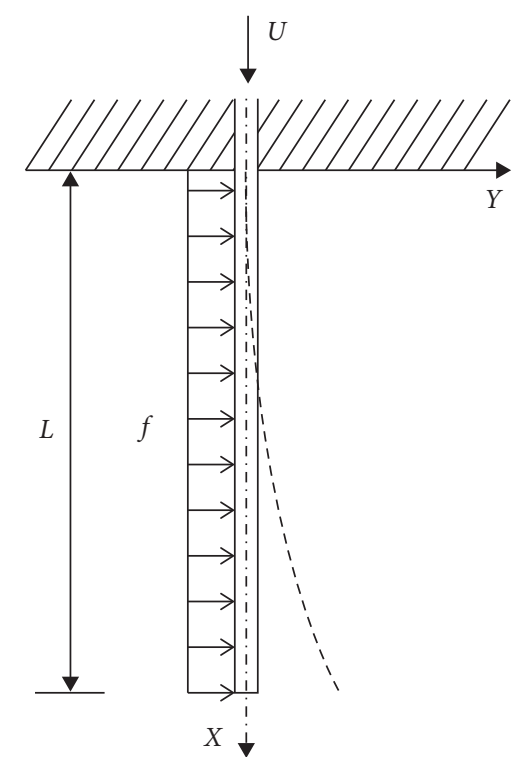

Figure 1: The dynamic model of a cantilevered pipe conveying pulsating fluid is given.

$$
\begin{aligned}
& (m+M) \ddot{y}+2 M U \dot{y}^{\prime}\left(1+y^{\prime 2}\right)+(m+M) g y^{\prime}\left(1+\frac{1}{2} y^{\prime 2}\right) \\
& +y^{\prime \prime}\left[M U^{2}\left(1+y^{\prime 2}\right)+(M \dot{U}-(m+M) g)(L-s)\right. \\
& \left.\cdot\left(1+\frac{1}{2} y^{\prime 2}\right)\right]+E I\left(y^{\prime \prime \prime \prime}+4 y^{\prime} y^{\prime \prime} y^{\prime \prime \prime}+y^{\prime \prime 3}+y^{\prime \prime \prime \prime} y^{\prime 2}\right) \\
& -y^{\prime \prime}\left[\int_{s}^{L} \int_{0}^{s}(m+M)\left(\dot{y}^{\prime 2}+y^{\prime} \ddot{y}^{\prime}\right) \mathrm{d} s \mathrm{~d} s\right. \\
& \left.+\int_{s}^{L}\left(\frac{1}{2} M \dot{U} y^{\prime 2}+2 M U y^{\prime} \dot{y}^{\prime}+M U^{2} y^{\prime} y^{\prime \prime}\right) \mathrm{d} s\right] \\
& +y^{\prime} \int_{0}^{s}(m+M)\left(\dot{y}^{\prime 2}+y^{\prime} \ddot{y}^{\prime}\right) \mathrm{d} s+f=0 .
\end{aligned}
$$

In this paper, we assume that the cantilevered pipe conveying pulsating fluid is made of Kelvin-Voigt type viscoelastic material. Therefore, we have

$$
\begin{aligned}
& \sigma=E \varepsilon+E \dot{\varepsilon} \\
& E=E\left(1+a \frac{\partial}{\partial t}\right) .
\end{aligned}
$$

In order to obtain the dimensionless governing equation of motion for the cantilevered pipe conveying pulsating fluid, the transformations of the variables and the parameters are introduced as

$$
\begin{aligned}
\xi & =\frac{s}{L}, \\
\eta & =\frac{y}{L}, \\
\tau & =\left(\frac{E I}{m+M}\right)^{1 / 2} \frac{t}{L^{2}}, \\
\alpha & =\left(\frac{E I}{m+M}\right)^{1 / 2} \frac{a}{L^{2}}, \\
U^{*} & =\left(\frac{M}{E I}\right)^{1 / 2} U L, \\
\gamma & =\frac{m+M}{E I} L^{3} g, \\
\beta & =\frac{m}{m+M}, \\
f^{*} & =\frac{L^{3}}{E I} f .
\end{aligned}
$$

For simplicity, the notation ${ }^{*}$ is omitted in the following analysis. We obtain the dimensionless partial differential governing equation of motion for the cantilevered pipe conveying pulsating fluid:

$$
\begin{aligned}
& \alpha \dot{\eta}^{\prime \prime \prime \prime}+\eta^{\prime \prime \prime \prime}+\ddot{\eta}+2 U \sqrt{\beta} \dot{\eta}^{\prime}\left(1+\eta^{\prime 2}\right)+\eta^{\prime \prime}\left[U^{2}\left(1+{\eta^{\prime}}^{2}\right)\right. \\
& \left.+(\dot{U} \sqrt{\beta}-\gamma)(1-\xi)\left(1+\frac{3}{2} \eta^{\prime 2}\right)\right] \\
& +\gamma \eta^{\prime}\left(1+\frac{1}{2} \eta^{\prime 2}\right)+\left(1+\alpha \frac{\partial}{\partial \tau}\right)\left(\eta^{\prime \prime \prime} \eta^{\prime 2}+4 \eta^{\prime} \eta^{\prime \prime} \eta^{\prime \prime \prime}+\eta^{\prime \prime 3}\right) \\
& -\eta^{\prime \prime}\left[\int_{\xi}^{1} \int_{0}^{\xi}\left(\eta^{\prime 2}+\eta^{\prime} \ddot{\eta}^{\prime}\right) \mathrm{d} \xi \mathrm{d} \xi+\int_{\xi}^{1}\left(\frac{1}{2} \dot{U} \sqrt{\beta} \eta^{\prime 2}\right.\right. \\
& \left.\left.+2 U \sqrt{\beta} \eta^{\prime} \dot{\eta}^{\prime 2}+U^{2} \eta^{\prime} \eta^{\prime \prime}\right) \mathrm{d} \xi\right] \\
& +\eta^{\prime} \int_{0}^{\xi}\left(\dot{\eta}^{\prime 2}+\eta^{\prime} \ddot{\eta}^{\prime}\right) d \xi+f=0 .
\end{aligned}
$$

It is assumed that the flow velocity $U$ is represented as a periodic perturbation $U=U_{0}+U_{1} \cos \Omega_{1} t$. In the meantime, the external excitation $f$ is described as a periodic perturbation $f=F+f_{1} \cos \Omega_{2} t$.

We find that there exist the gyroscopic terms in equation (4), which means that the damping of the cantilevered pipe conveying pulsating fluid is too large to ignore. The traditional methods dealing with the nonlinear oscillations are first discretized by Galerkin method and then are analyzed by the perturbation method. However, these methods usually cancel the coefficients related to the gyroscopic terms and lose a lot of important information. In order to retain the gyroscopic terms as much as possible, we use a combining method of both the multiple-scale method and Galerkin method to obtain the average equation of the cantilevered pipe conveying pulsating fluid. 
For the perturbation analysis, equation (4) is simplified and the following expression is given for the cantilevered pipe conveying pulsating fluid:

$$
\begin{aligned}
M \ddot{\eta}+G \dot{\eta}+K \eta= & \varepsilon F(\eta)-\varepsilon N(\eta) \\
& -2 \varepsilon U_{1} \sqrt{\beta} \dot{\eta}^{\prime} \cos \Omega_{1} t-2 \varepsilon \eta^{\prime \prime} U_{0} U_{1} \cos \Omega_{1} t \\
& -\varepsilon \eta^{\prime \prime} \Omega_{1} U_{1} \sqrt{\beta}(1-\xi) \sin \Omega_{1} t,
\end{aligned}
$$

where $\varepsilon$ represents the small-scale transformation and other parameters are given as follows:

$$
\begin{aligned}
M= & I \\
G= & 2 U_{0} \sqrt{\beta} \frac{\partial}{\partial x}+\alpha \frac{\partial}{\partial x^{4}}, \\
K= & {\left[U_{0}^{2}-\gamma(1-\xi)\right] \frac{\partial}{\partial x^{2}}+\gamma \frac{\partial}{\partial x}+\frac{\partial}{\partial x^{4}}, } \\
F(\eta)= & -F-f_{1} \cos \Omega_{2} t, \\
N(\eta)= & 2 U_{0} \sqrt{\beta} \dot{\eta}^{\prime} \eta^{\prime 2}+\eta^{\prime \prime} \eta^{\prime 2}\left[U_{0}^{2}-\frac{3}{2} \gamma(1-\xi)\right]+\frac{1}{2} \gamma \eta^{\prime 3} \\
& +\left(1+a \frac{\partial}{\partial \tau}\right)\left(\eta^{\prime \prime \prime \prime} \eta^{\prime 2}+4 \eta^{\prime} \eta^{\prime \prime} \eta^{\prime \prime \prime}+\eta^{\prime \prime 3}\right) \\
& -\eta^{\prime \prime} \int_{\xi}^{1} \int_{0}^{\xi}\left(\eta^{\prime 2}+\eta^{\prime} \ddot{\eta}^{\prime}\right) \mathrm{d} \xi \mathrm{d} \xi \\
& -\eta^{\prime \prime} \int_{\xi}^{1}\left(2 U_{0} \sqrt{\beta} \eta^{\prime} \dot{\eta}^{\prime 2}+U_{0}^{2} \eta^{\prime} \eta^{\prime \prime}\right) \mathrm{d} \xi \\
& +\eta^{\prime} \int_{0}^{\xi}\left(\dot{\eta}^{\prime 2}+\eta^{\prime} \ddot{\eta}^{\prime}\right) \mathrm{d} \xi .
\end{aligned}
$$

The method of multiple scales [47] is applied to the partial differential equation (5) to obtain the uniform solutions in the following form:

$$
\eta(t, \varepsilon)=\eta_{0}\left(T_{0}, T_{1}\right)+\varepsilon \eta_{1}\left(T_{0}, T_{1}\right)
$$

where $T_{0}=t$ and $T_{1}=\varepsilon t$.

Then, the derivatives with respect to $t$ become

$$
\begin{aligned}
\frac{\mathrm{d}}{\mathrm{d} t} & =\frac{\partial}{\partial T_{0}} \frac{\partial T_{0}}{\partial t}+\frac{\partial}{\partial T_{1}} \frac{\partial T_{1}}{\partial t}+\cdots=\frac{\partial}{\partial T_{0}}+\varepsilon \frac{\partial}{\partial T_{1}}+\cdots \\
& =D_{0}+\varepsilon D_{1}+\cdots \\
\frac{\mathrm{d}^{2}}{\mathrm{~d} t^{2}} & =\left(D_{0}+\varepsilon D_{1}+\cdots\right)^{2}=D_{0}^{2}+2 \varepsilon D_{0} D_{1}+\varepsilon^{2} D_{1}^{2}+\cdots,
\end{aligned}
$$

where $D_{0}=\left(\partial / \partial T_{0}\right)$ and $D_{1}=\left(\partial / \partial T_{1}\right)$.

Substituting equations (7), (8a), and (8b) into equations (6a)-(6c) and eliminating the secular terms, we obtain the following:

$\varepsilon^{0}:$

$$
M D_{0}^{2} \eta_{0}+G\left(D_{0} \eta_{0}\right)+K \eta_{0}=0
$$

$\mathcal{E}^{1}$ :

$$
\begin{aligned}
M D_{0}^{2} \eta_{1}+G D_{0} \eta_{1}+K \eta_{1}= & -F-f_{1} \cos \Omega_{2} t \\
& -2 U_{0} \sqrt{\beta} \frac{\partial^{2} \eta_{0}}{\partial T_{0} \partial X}\left(\frac{\partial \eta_{0}}{\partial X}\right)^{2}+\frac{\partial^{2} \eta_{0}}{\partial X^{2}}\left(\frac{\partial \eta_{0}}{\partial X}\right)^{2}\left(U_{0}^{2}-\frac{3}{2} \gamma(1-\xi)\right)-\frac{1}{2} \gamma\left(\frac{\partial \eta_{0}}{\partial X}\right)^{3} \\
& -\left(1+\alpha \frac{\partial}{\partial T_{0}}\right)\left[\frac{\partial^{4} \eta_{0}}{\partial X^{4}}\left(\frac{\partial \eta_{0}}{\partial X}\right)^{2}+4 \frac{\partial \eta_{0}}{\partial X} \frac{\partial^{2} \eta_{0}}{\partial X^{2}} \frac{\partial^{3} \eta_{0}}{\partial X^{3}}+\left(\frac{\partial^{2} \eta_{0}}{\partial X^{2}}\right)^{3}\right] \\
& +\frac{\partial^{2} \eta_{0}}{\partial X^{2}}\left[\int_{\xi}^{1} \int_{0}^{\xi}\left(\left(\frac{\partial \eta_{0}}{\partial X}\right)^{2}+\frac{\partial \eta_{0}}{\partial X} \frac{\partial^{3} \eta_{0}}{\partial T_{0}^{2} \partial X}\right) \mathrm{d} \xi \mathrm{d} \xi+\int_{0}^{\xi}\left(2 U_{0} \sqrt{\beta} \frac{\partial \eta_{0}}{\partial X} \frac{\partial^{2} \eta_{0}}{\partial T_{0} \partial X}+U_{0}^{2} \frac{\partial \eta_{0}}{\partial X} \frac{\partial^{2} \eta_{0}}{\partial X^{2}}\right) \mathrm{d} \xi\right] \\
& -\frac{\partial \eta_{0}}{\partial X} \int_{0}^{\xi}\left(\frac{\partial^{2} \eta_{0}}{\partial T_{0} \partial X}+\frac{\partial \eta_{0}}{\partial X} \frac{\partial^{3} \eta_{0}}{\partial T_{0}^{2} \partial X}\right) \mathrm{d} \xi-2 U_{1} \sqrt{\beta} \frac{\partial^{2} \eta_{0}}{\partial T_{0} \partial X} \cos \Omega_{1} t \\
& -\frac{\partial^{2} \eta_{0}}{\partial X^{2}}\left[2 U_{0} U_{1} \cos \Omega_{1} t-\Omega_{1} U_{1} \sqrt{\beta}(1-\xi) \sin \Omega_{1} t\right]-2 D_{0} D_{1} \eta_{0}-G D_{1} \eta_{0} .
\end{aligned}
$$


The boundary conditions are given as

$$
\begin{gathered}
y(0)=y^{\prime}(0)=0, \\
y^{\prime \prime}(L)=y^{\prime \prime \prime}(L)=0 .
\end{gathered}
$$

In order to simplify the cantilevered pipe conveying pulsating fluid to the finite dimension by using Galerkin discretization, the modal function is selected as a beam function:

$$
\varphi_{j}(\xi)=\cosh \left(\beta_{j} x\right)-\cos \left(\beta_{j} x\right)-\lambda_{j}\left[\sin h\left(\beta_{j} x\right)-\sin \left(\beta_{j} x\right)\right] .
$$
follows:

The general solution of equation (9) is expressed as

$$
\eta\left(T_{0}, T_{1}, \xi\right)=\sum_{j=0}^{N} \varphi_{j}(\xi) A_{j}\left(T_{1}\right) e^{i \omega_{j} T_{0}}+c c,
$$

where we take the nonlinear oscillations of the cantilevered pipe conveying pulsating fluid into account in the first two oscillation modes:

$$
\eta\left(T_{0}, T_{1}, x\right)=\varphi_{1}(x) A_{1}\left(T_{1}\right) e^{i \omega_{1} T_{0}}+\varphi_{3}(x) A_{2}\left(T_{1}\right) e^{i \omega_{3} T_{0}}+c c .
$$

Substituting equation (14) into equation (5) and after a series of calculations and simplification, the approximate frequency relationships are obtained as follows:

$$
\begin{aligned}
& \omega_{1}^{2}=\left(\alpha_{12}-\frac{\alpha_{11} \widetilde{L}}{2}\right) \gamma+\alpha_{11} U\left(U+\frac{\widetilde{L} \sqrt{\beta}}{2}\right), \\
& \omega_{3}^{2}=\left(\alpha_{22}-\frac{\alpha_{21} \widetilde{L}}{2}\right) \gamma+\alpha_{21} U\left(U+\frac{\widetilde{L} \sqrt{\beta}}{2}\right),
\end{aligned}
$$

where $\widetilde{L}, \beta$, and $U$, respectively, represent the length, mass ratio, and flow velocity, and $\alpha_{11}, \alpha_{12}, \alpha_{21}, \alpha_{22}$, and $\gamma$ are the system parameters and dimensionless parameters.

According to the geometries and the material properties of the cantilevered pipe conveying pulsating fluid, the parameters are chosen as $\beta=0.9, U=3, \alpha_{11}=-1.9, \alpha_{12}=11$, $\alpha_{21}=-4.8, \alpha_{22}=30$, and $\gamma=2$. The length $\widetilde{L}$ is selected as the variable. The first-order and third-order natural frequencies are solved, as shown in the Campbell diagram in Figure 2. We find that the natural frequencies of the firstorder and third-order oscillation modes for the cantilevered pipe conveying fluid decrease as the length increases. It is observed that $1: 3$ internal resonance happens in the cantilevered pipe conveying pulsating fluid.

We only consider the case of $1: 3$ internal resonance, principle parameter resonance, and $1 / 2$ subharmonic resonance for equation (5). In this resonant case, there exist the following relations:

$$
\begin{aligned}
& \omega_{1}=\frac{1}{2} \Omega_{1}+\varepsilon \sigma_{1}, \\
& \omega_{3}=\frac{3}{2} \Omega_{1}+\varepsilon \sigma_{2}, \\
& \Omega_{1}=\frac{1}{2} \Omega_{2},
\end{aligned}
$$

where $\sigma_{1}$ and $\sigma_{2}$ are two detuning parameters.

Substituting equations (12), (14), and (16) into equation (10) yields

$$
\begin{aligned}
M D_{0}^{2} \eta_{1}+G D_{0} \eta_{1}+K \eta_{1}= & \Gamma_{1} e^{i T_{0}\left((1 / 2) \Omega_{1}+\varepsilon \sigma_{1}\right)}+\Gamma_{2} e^{i T_{0}\left((3 / 2) \Omega_{1}+\varepsilon \sigma_{2}\right)}-q_{1} e^{(1 / 2) i T_{0} \Omega_{1}} \\
& +\Gamma_{3} e^{i T_{0}\left((1 / 2) \Omega_{1}-\varepsilon \sigma_{1}\right)}+\Gamma_{4} e^{i T_{0}\left((1 / 2) \Omega_{1}+\varepsilon \sigma_{2}\right)}+\Gamma_{5} e^{i T_{0}\left((3 / 2) \Omega_{1}+\varepsilon \sigma_{1}\right)}+c c+\text { NST, }
\end{aligned}
$$

where

$$
\begin{aligned}
\Gamma_{1}= & i \Omega A_{11}^{2} A_{12}\left(U_{0} \sqrt{\beta} m_{1}+\frac{3}{2} \alpha m_{2}\right)+i \Omega A_{11} A_{21} A_{22}\left(2 U_{0} \sqrt{\beta} m_{3}+\frac{3}{2} \alpha m_{4}\right) \\
& +A_{11}^{2} A_{12}\left(3 U_{0}^{2} m_{5}-\gamma m_{6}-3 m_{7}+\frac{3}{4} \Omega^{2} m_{8}\right) \\
& +A_{11} A_{21} A_{22}\left(U_{0}^{2} m_{9}-\gamma m_{10}+m_{4}+\Omega^{2} m_{11}\right)-i \Omega_{1} \dot{A}_{11} M \varphi_{1}-\dot{A}_{11} G \varphi_{1}, \\
\Gamma_{2}= & i \Omega A_{21}^{2} A_{22}\left(3 U_{0} \sqrt{\beta} n_{1}+\alpha n_{2}\right)+i \Omega A_{11} A_{12} A_{21}\left(6 U_{0} \sqrt{\beta} n_{3}+\alpha n_{4}\right) \\
& +A_{21}^{2} A_{22}\left(3 U_{0}^{2} n_{5}-\gamma n_{6}+n_{7}+\Omega^{2} n_{8}\right) \\
& +A_{11} A_{12} A_{21}\left(U_{0}^{2} n_{9}-\gamma n_{10}+n_{4}+\Omega^{2} n_{11}\right)-3 i \Omega_{1} \dot{A}_{21} M \varphi_{2}-\dot{A}_{21} G \varphi_{2},
\end{aligned}
$$




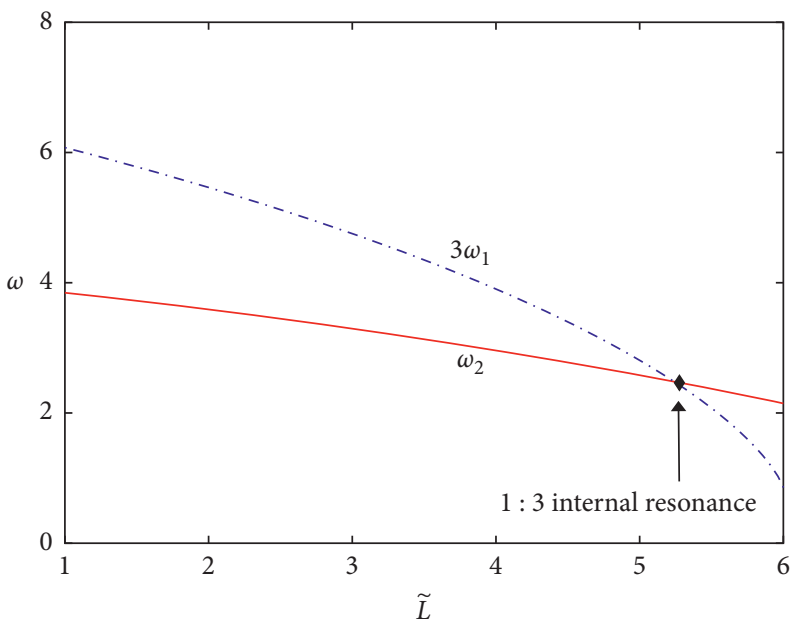

Figure 2: The Campbell diagram of the cantilevered pipe conveying pulsating fluid is obtained in the case of $1: 3$ internal resonance.

$$
\begin{aligned}
\Gamma_{3}= & A_{11} A_{12}^{2}\left(-\frac{1}{2} \Omega_{1}^{2} m_{12}-i \Omega_{1} U_{0} \sqrt{\beta} m_{13}+U_{0}^{2} m_{14}\right)+i \Omega A_{11} A_{12} A_{21}\left(6 U_{0} \sqrt{\beta} n_{3}+\alpha n_{4}\right) \\
& +A_{11} A_{21} A_{22}\left(-\Omega_{1}^{2} m_{15}-i \Omega_{1} U_{0} \sqrt{\beta} m_{16}+U_{0}^{2} m_{17}\right)+\frac{1}{2} i U_{1} \sqrt{\beta} A_{12} \Omega_{1} m_{18}-U_{0} U_{1} A_{12} m_{19}, \\
\Gamma_{4}= & \frac{1}{2} i A_{21} \Omega_{1} U_{1} \sqrt{\beta} m_{20}-A_{21} U_{0} U_{1} m_{21}, \\
\Gamma_{5}= & \frac{1}{2} i A_{11} \Omega_{1} U_{1} \sqrt{\beta} n_{12}-A_{11} U_{0} U_{1} n_{13} .
\end{aligned}
$$

The solution exists when the nonhomogeneous equation corresponding to equation (17) satisfies that the right side of the equation is orthogonal to the solution of its homogeneous accompanying equation. Thus, we have

$$
\begin{aligned}
& \int_{0}^{1}\left(\Gamma_{1}-f_{1} e^{-i \sigma_{1} T_{1}}+\Gamma_{3} e^{-2 i \sigma_{1} T_{1}}+\Gamma_{4} e^{-i T_{1}\left(\sigma_{1}-\sigma_{2}\right)}\right) \bar{\varphi}_{1} \mathrm{~d} x=0 \\
& \int_{0}^{1}\left(\Gamma_{2}+\Gamma_{5} \mathrm{e}^{-i T_{1}\left(\sigma_{2}-\sigma_{1}\right)}\right) \bar{\varphi}_{2} \mathrm{~d} x=0
\end{aligned}
$$

Let $A_{1}$ and $A_{2}$ be of the following forms:

$$
\begin{aligned}
& A_{1}\left(T_{1}\right)=\frac{1}{2}\left(a_{1} e^{i \varphi_{1}}\right) \cdot e^{-i\left(\sigma_{1} T_{1}-2 m \pi\right)}, \\
& A_{2}\left(T_{1}\right)=\frac{1}{2}\left(a_{2} e^{i \varphi_{2}}\right) \cdot e^{-i\left(\sigma_{2} T_{1}-2 n \pi\right)} .
\end{aligned}
$$

Substituting equation (20) into equations (19a) and (19b) and separating the real and imaginary parts, the averaged equations in the polar form are obtained as follows:

$$
\begin{aligned}
\dot{a}_{1}= & \frac{1}{8}\left(\beta_{14}+\beta_{111}-\beta_{10} \cos 2 \varphi_{1}+\beta_{16} \sin 2 \varphi_{1}\right) a_{1} a_{2}^{2}+\frac{1}{2}\left(\beta_{17} \sin 2 \varphi_{1}-\beta_{18} \cos 2 \varphi_{1}\right) a_{1} \\
& +\frac{1}{8}\left(\beta_{13}+\beta_{110}\right) a_{1}^{3}+\frac{1}{2}\left[\beta_{19} \sin \left(\varphi_{1}-\varphi_{2}\right)-\beta_{20} \cos \left(\varphi_{1}-\varphi_{2}\right)\right] a_{2}-F \sin \varphi_{1}-\mu a_{1}, \\
a_{1} \dot{\varphi}_{1}= & \frac{1}{8}\left(\beta_{12}+\beta_{10} \sin 2 \varphi_{1}+\beta_{16} \cos 2 \varphi_{1}\right) a_{1} a_{2}^{2}+\frac{1}{2}\left(\beta_{17} \cos 2 \varphi_{1}+\beta_{18} \sin 2 \varphi_{1}\right) a_{1} \\
& +\frac{1}{8}\left(\beta_{11}-\beta_{15}\right) a_{1}^{3}+\frac{1}{2}\left[\beta_{19} \cos \left(\varphi_{1}-\varphi_{2}\right)+\beta_{20} \sin \left(\varphi_{1}-\varphi_{2}\right)\right] a_{2}-F \cos \varphi_{1}-\sigma_{1} a_{1}, \\
\dot{a}_{2}= & \frac{1}{8} \beta_{23} a_{2}^{3}+\frac{1}{8} \beta_{24} a_{2} a_{1}^{2}+\frac{1}{2}\left[\beta_{25} \sin \left(\varphi_{1}-\varphi_{2}\right)+\beta_{26} \cos \left(\varphi_{1}-\varphi_{2}\right)\right] a_{1}-\mu a_{2}, \\
a_{2} \dot{\varphi}_{2}= & \frac{1}{8} \beta_{21} a_{2}^{3}+\frac{1}{8} \beta_{22} a_{2} a_{1}^{2}+\frac{1}{2}\left[\beta_{25} \cos \left(\varphi_{1}-\varphi_{2}\right)+\beta_{26} \sin \left(\varphi_{1}-\varphi_{2}\right)\right] a_{1}-\sigma_{2} a_{2} .
\end{aligned}
$$


In order to obtain the averaged equations in the Cartesian form, we express $A_{1}$ and $A_{2}$ in the following forms:

$$
\begin{aligned}
& A_{1}\left(T_{1}\right)=\left[x_{1}\left(T_{1}\right)-i x_{2}\left(T_{1}\right)\right] e^{-i\left(o_{1} T_{1}-2 m \pi\right)}, \\
& A_{2}\left(T_{1}\right)=\left[x_{3}\left(T_{1}\right)-i x_{4}\left(T_{1}\right)\right] e^{-i\left(o_{2} T_{1}-2 n \pi\right)} .
\end{aligned}
$$

Based on the same way as the aforementioned analysis, the averaged equations in the Cartesian form are obtained for the cantilevered pipe conveying pulsating fluid:

$$
\begin{aligned}
\dot{x}_{1}= & \left(\beta_{17}-\mu\right) x_{1}-\left(\beta_{18}+\sigma_{1}\right) x_{1}+\beta_{19} x_{3}+\beta_{20} x_{4}+\left(\beta_{11}-\beta_{15}\right) x_{1}\left(x_{1}^{2}+x_{2}^{2}\right)+\left(\beta_{12}-\beta_{16}\right) x_{1}\left(x_{3}^{2}+x_{4}^{2}\right) \\
& -\left(\beta_{13}+\beta_{110}-p_{15}\right) x_{2}\left(x_{1}^{2}+x_{2}^{2}\right)-\left(\beta_{14}+\beta_{111}-2 p_{17}\right) x_{2}\left(x_{3}^{2}+x_{4}^{2}\right), \\
\dot{x}_{2}= & \left(\beta_{17}-\mu\right) x_{2}-\left(\beta_{18}-\sigma_{1}\right) x_{1}-\beta_{19} x_{4}+\beta_{20} x_{3}+\left(\beta_{11}+\beta_{15}\right) x_{2}\left(x_{1}^{2}+x_{2}^{2}\right)+\left(\beta_{12}+\beta_{16}\right) x_{2}\left(x_{3}^{2}+x_{4}^{2}\right) \\
& -\left(\beta_{13}-\beta_{110}-p_{15}\right) x_{1}\left(x_{1}^{2}+x_{2}^{2}\right)-\left(\beta_{14}-\beta_{111}-2 p_{17}\right) x_{1}\left(x_{3}^{2}+x_{4}^{2}\right)-F p_{0}, \\
\dot{x}_{3}= & -\mu x_{3}-\sigma_{2} x_{4}+\beta_{25} x_{1}+\beta_{26} x_{2}+\beta_{21} x_{3}\left(x_{3}^{2}+x_{4}^{2}\right)+\beta_{22} x_{3}\left(x_{1}^{2}+x_{2}^{2}\right) \\
& -\beta_{23} x_{4}\left(x_{3}^{2}+x_{4}^{2}\right)-\beta_{24} x_{4}\left(x_{1}^{2}+x_{2}^{2}\right), \\
\dot{x}_{4}= & -\mu x_{4}+\sigma_{2} x_{3}-\beta_{25} x_{2}+\beta_{26} x_{1}+\beta_{21} x_{4}\left(x_{3}^{2}+x_{4}^{2}\right)+\beta_{22} x_{4}\left(x_{1}^{2}+x_{2}^{2}\right) \\
& +\beta_{23} x_{3}\left(x_{3}^{2}+x_{4}^{2}\right)+\beta_{24} x_{3}\left(x_{1}^{2}+x_{2}^{2}\right),
\end{aligned}
$$

where the coefficients are given, respectively, as

$$
\begin{aligned}
& \beta_{10}=\Omega_{1} U_{0} \sqrt{\beta} p_{16} \\
& \beta_{11}=U_{0} \sqrt{\beta} p_{1}+\frac{3}{2} \alpha p_{2} \\
& \beta_{12}=2 U_{0} \sqrt{\beta} p_{3}+\frac{3}{2} \alpha p_{4} \\
& \beta_{13}=3 U_{0}^{2} p_{5}-\gamma p_{6}+3 p_{7}+\frac{3}{4} \Omega^{2} p_{8} \\
& \beta_{14}=U_{0}^{2} p_{9}-\gamma p_{10}+p_{4}+\Omega^{2} p_{11} \\
& \beta_{15}=\Omega_{1} U_{0} \sqrt{\beta} p_{13} \\
& \beta_{16}=U_{0}^{2} p_{17}-\Omega^{2} p_{15} \\
& \beta_{17}=\frac{1}{2} U_{1} \Omega_{1} \sqrt{\beta} p_{18} \\
& \beta_{111}=U_{0}^{2} p_{17}-\Omega^{2} p_{15} \\
& \beta_{20}=U_{0} U_{1} p_{21} \\
& \beta_{110}=\frac{1}{2} \Omega_{19} U_{1} \sqrt{\beta} p_{20} \\
& \beta_{0}-\frac{1}{2} \Omega^{2} p_{12} \\
& \beta_{10} \\
& \beta_{10} \\
& \beta_{10}
\end{aligned}
$$

$$
\begin{aligned}
& \beta_{21}=\Omega\left(3 U_{0} \sqrt{\beta} q_{1}+\alpha q_{2}\right), \\
& \beta_{22}=\Omega\left(6 U_{0} \sqrt{\beta} q_{3}+\alpha q_{4}\right), \\
& \beta_{23}=3 U_{0}^{2} q_{5}-\gamma q_{6}+q_{7}+\Omega^{2} q_{8}, \\
& \beta_{24}=U_{0}^{2} q_{9}-\gamma q_{10}+q_{4}+\Omega^{2} q_{11}, \\
& \beta_{25}=\frac{1}{2} \Omega_{1} U_{1} \sqrt{\beta} q_{12}, \\
& \beta_{26}=U_{0} U_{1} q_{13} .
\end{aligned}
$$

Equations (21a)-(21d) and (23a)-(23d) describe the nonlinear oscillations of the cantilevered pipe conveying pulsating fluid under the external harmonic excitation. It is known that the constant solutions of the averaged equation correspond to the periodic solutions of the original system, the periodic solutions of the averaged equation correspond to the quasi-periodic solutions of the original system, and the chaotic solutions of the averaged equation correspond to the chaotic solutions of the original system.

\section{Analysis of Resonant Responses}

The resonant response curves are the important basis for judging and studying the nonlinear oscillations. They include many complex nonlinear dynamic phenomena. The practical problems can be solved better by analyzing these phenomena. Based on the averaged equations (21a)-(21d) in the polar form, we analyze the amplitude-frequency response curves to reveal the nonlinear oscillations of the cantilevered pipe conveying pulsating fluid. Since $\varphi_{1}$ and $\varphi_{2}$ are all constant, we make $\varphi_{1}=\varphi_{2}=0.25 \pi$. Therefore, we 
obtain the amplitude-frequency response functions of the cantilevered pipe conveying pulsating fluid:

$$
\begin{gathered}
{\left[\frac{1}{8}\left(\beta_{13}+\beta_{110}\right) a_{1}^{3}+\frac{1}{8}\left(\beta_{14}+\beta_{111}\right) a_{1} a_{2}^{2}-\frac{1}{2} \beta_{20} a_{2}-\frac{\sqrt{2}}{2} F-\mu a_{1}\right]^{2}} \\
+\left[\frac{1}{8}\left(\beta_{11}+\beta_{15}\right) a_{1}^{3}+\frac{1}{8} \beta_{12} a_{1} a_{2}^{2}+\frac{1}{2}\left(\beta_{19}-\beta_{20}\right) a_{2}-\frac{\sqrt{2}}{2} F-\sigma_{1} a_{1}\right]^{2} \\
=\left(\frac{1}{8} \beta_{110} a_{1} a_{2}^{2}-\frac{1}{2} \beta_{18} a_{1}\right)^{2}+\left(\frac{1}{8} \beta_{16} a_{1} a_{2}^{2}+\frac{1}{2} \beta_{17} a_{1}\right)^{2} \\
\left(\frac{1}{8} \beta_{24} a_{2} a_{1}^{2}+\frac{1}{8} \beta_{23} a_{2}^{3}\right)^{2}+\left(\frac{1}{8} \beta_{22} a_{2} a_{1}^{2}+\frac{1}{8} \beta_{21} a_{2}^{3}\right)^{2} \\
=\left(\frac{1}{2} \beta_{26} a_{1}+\mu a_{2}\right)^{2}+\left(\frac{1}{2} \beta_{25} a_{1}+\sigma_{2} a_{2}\right)^{2}
\end{gathered}
$$

Two cases of the amplitude-frequency response curves $[48,49]$ are considered for the cantilevered pipe conveying pulsating fluid:

(1) There is no coupling effect between the first-order and third-order oscillation modes. We only consider the decoupled case and set $a_{2}=0$ in equation (25a) and $a_{1}=0$ in equation (25b).

(2) We let an amplitude change when another is fixed because the amplitude is much smaller than that in the case of weak coupling; namely, set $a_{2}=1$ in equation (25a) and $a_{1}=1$ in equation (25b).

We obtain the amplitude-frequency response curves in the decoupled and coupled cases based on equations (25a) and (25b). Based on the geometries and the material properties of the cantilevered pipe conveying pulsating fluid, the basic parameters are chosen as

$$
\begin{aligned}
U_{0} & =1.5, \\
F & =2.5, \\
\beta & =0.9, \\
\gamma & =2, \\
\mu & =4, \\
U_{1} & =3.5, \\
\Omega & =1.2, \\
\Omega_{1} & =10, \\
p_{1} & =2 \times 10^{3}, \\
p_{2} & =4, \\
p_{3} & =2, \\
p_{4} & =8, \\
p_{5} & =10, \\
p_{6} & =2, \\
p_{7} & =-20, \\
p_{8} & =1,
\end{aligned}
$$

$$
\begin{aligned}
p_{9} & =100, \\
p_{10} & =-1, \\
p_{11} & =-3, \\
p_{12} & =-14, \\
p_{13} & =-6, \\
p_{14} & =-10, \\
p_{15} & =14, \\
p_{16} & =10, \\
p_{17} & =4, \\
p_{18} & =-1, \\
p_{19} & =20, \\
p_{20} & =30, \\
p_{21} & =12, \\
p_{22} & =1, \\
p_{23} & =1, \\
p_{24} & =6, \\
q_{1} & =15, \\
q_{2} & =16, \\
q_{3} & =-4.7, \\
q_{4} & =18, \\
q_{5} & =18, \\
q_{6} & =7, \\
q_{12} & =4, \\
q_{13} & =0.25
\end{aligned}
$$

For both the decoupled and coupled cases, when the velocity parametric excitation is $U_{0}=1.5$ and external excitation is $F=2.5$, it is found that the cantilevered pipe conveying pulsating fluid has the hardening spring characteristics because the amplitude-frequency response curves bend to right, as shown in Figures 3(a) and 3(b). The blue line represents the coupled case and the red line gives the decoupled case. The bending of the amplitude-frequency response curves also leads to the multiple amplitudes and bifurcations. The multiple amplitudes mean that there are several possible responses in the cantilevered pipe conveying pulsating fluid. The actual response among several possible responses depends on the initial conditions. Hence, the typical jumping phenomena of the nonlinear oscillations can be observed for the cantilevered pipe conveying pulsating fluid. Comparing the amplitude-frequency response curves between two cases given in Figure 3(a), we find that the amplitude of the coupled case is larger and the resonant response interval is wider than the decoupled case. The coupling effect of two oscillation modes will not change the 


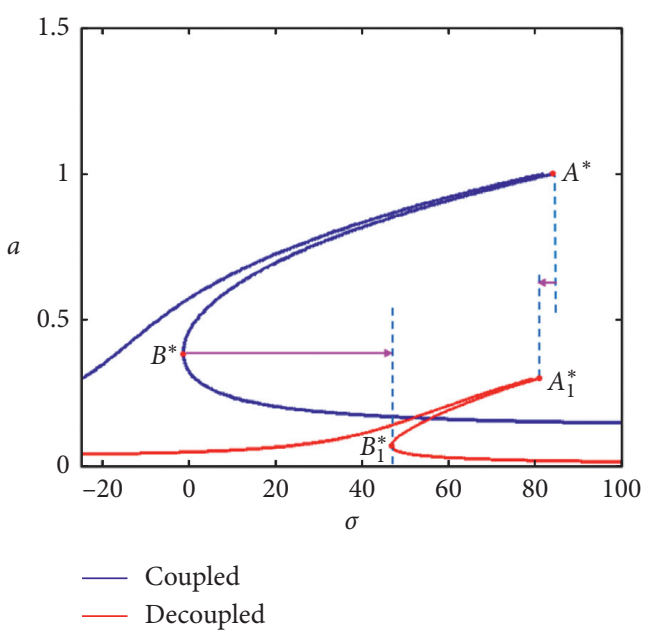

(a)

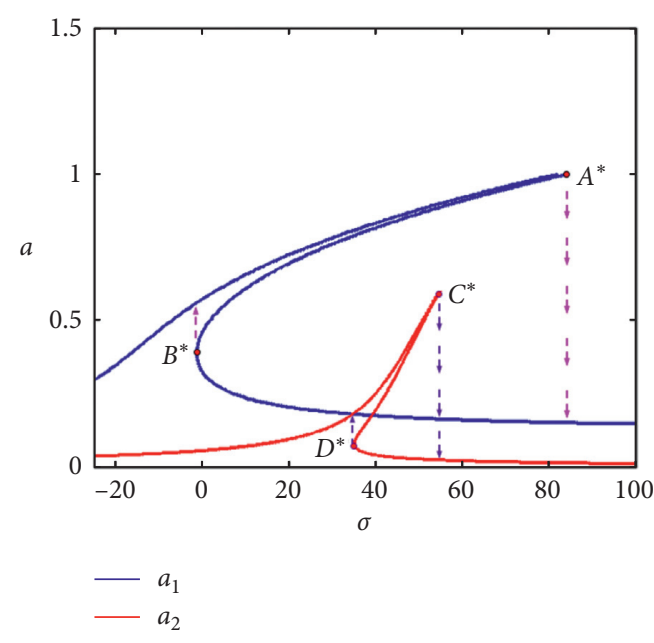

(b)

Figure 3: The amplitude-frequency response curves of the cantilevered pipe conveying pulsating fluid are obtained when flow velocity is $U_{0}=1.5$ and external force is $F=2.5$. (a) Coupling influence on amplitude-frequency response curves; (b) amplitude-frequency response curves of the first-order and third-order modes.

system's stiffness but will cause a stronger nonlinear resonant response. Furthermore, it can be also observed that the amplitude of the first-order mode is larger than that of the third-order mode in the coupled case, as shown in Figure 3(b). In the coupled case, there exists the mode interaction between two oscillation modes. Therefore, the amplitude-frequency response curves of the coupled case are considered for the cantilevered pipe conveying pulsating fluid in the following analysis.

In the weak coupling cases, the influences of the flow velocity $U_{0}$, external excitation $F$, and mass parameter $\beta$ on the amplitude-frequency response curves are analyzed in Figures 4(a)-4(c), where the mass parameter $\beta$ denotes the ratio of the pipe mass and total mass. Figure $4(\mathrm{a})$ illustrates the amplitude-frequency response curves of the cantilevered pipe conveying pulsating fluid when the flow velocities are $U_{0}=1.5$ and $U_{0}=2.0$, respectively. With the increases of the flow velocity $U_{0}$, it is obviously found that the amplitude is larger and the resonant response interval is wider for the cantilevered pipe conveying pulsating fluid, as shown in Figure 4(a). The increase of flow velocity will not change the system's stiffness but will cause a stronger nonlinear resonant response. These characteristics indicate that the cantilevered pipe conveying pulsating fluid obtains the more external energy. Figure 4(b) gives the amplitude-frequency response curves of the cantilevered pipe conveying pulsating fluid when the external excitations are $F=2.5$ and $F=7.0$, respectively. In Figure 4(b), we find that the increase of the external excitation $F$ mainly results in the larger amplitude, which has little effect on the resonant response interval. Figure 4(c) shows the amplitude-frequency response curves of the cantilevered pipe conveying pulsating fluid when the mass parameters are $\beta=0.9$ and $\beta=0.7$, respectively. In Figure $4(\mathrm{c})$, as the mass parameter $\beta$ decreases, which means that the pipe mass is lighter, we find that the nonlinear stiffness of the cantilevered pipe conveying pulsating fluid does not change. However, it is observed that the resonance of the cantilevered pipe conveying pulsating fluid is more easily excited and the amplitudes of the nonlinear oscillations are greater when mass parameters are smaller.

The effects of the flow velocity and external excitation on the amplitude-frequency response curves are investigated for the cantilevered pipe conveying pulsating fluid in Figures 5(a) and 5(b) under the coupling cases. We set $\sigma=10$ and other parameters are the same as the parameters given in Figures 3 and 4. The amplitude-frequency response curves of the flow velocity are given in Figure 5(a). It can be shown that the amplitudes of the cantilevered pipe conveying pulsating fluid increase with the increase of the flow velocity. Thus, the energy of the fluid motion is transferred into the energy of the cantilevered pipe. The fluid-structure interaction occurs in the cantilevered pipe conveying pulsating fluid. There are two jumping phenomena on the amplitude-frequency response curves with the increase of the flow velocity: one is jumping up and one is jumping down. The oscillations of the cantilevered pipe conveying pulsating fluid are stable at a lower amplitude. The increase and decrease of the amplitudes alternatively change with the increase of the flow velocity. It is indicated that the flow velocity will affect the nonlinear oscillation behaviors of the cantilevered pipe conveying pulsating fluid in a certain range. The amplitude-frequency response curves of the external excitation are given in Figure 5(b). The amplitudes of the cantilevered pipe conveying pulsating fluid become larger with the gradual increase of the external excitation. The sudden increase in the amplitude of the cantilevered pipe conveying pulsating fluid appears when a jumping phenomenon occurs. It is illustrated that the cantilevered pipe conveying pulsating fluid will obtain more energy to create the larger amplitude with the increase of the external excitation.

We explore the influences of different parameters on the amplitude-frequency and force-amplitude response curves in the cantilevered pipe conveying pulsating fluid, respectively. In 


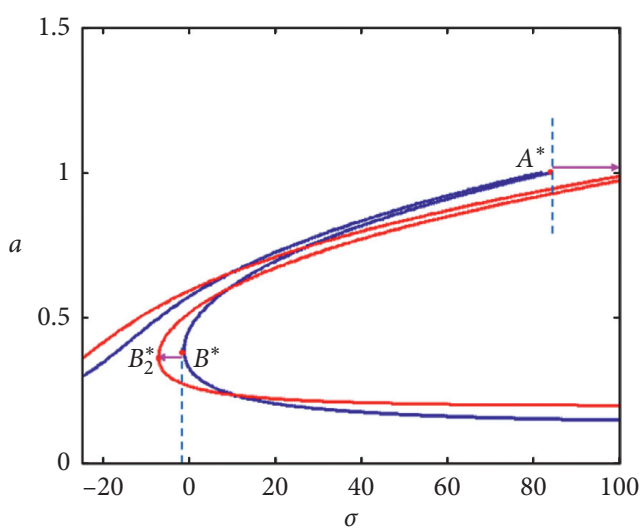

$U_{0}=1.5$
$U_{0}=2$

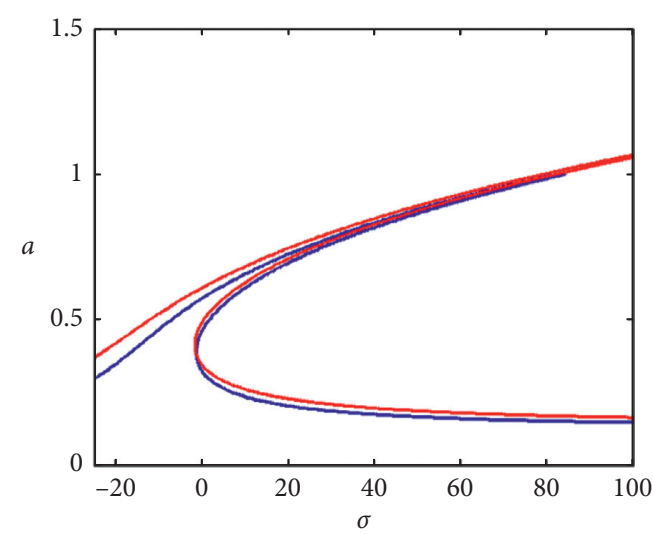

$-F=2.5$

$-F=7$

(a)

(b)

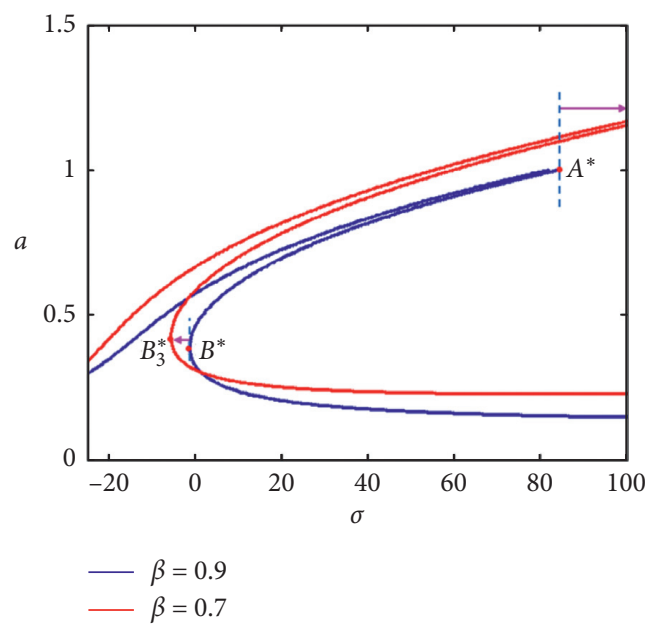

(c)

FIGURE 4: The amplitude-frequency response curves of the cantilevered pipe conveying pulsating fluid are obtained in the coupling case with different parameters. (a) Amplitude-frequency response curves when flow velocities $U_{0}=1.5$ and $U_{0}=2.0$, (b) amplitude-frequency response curves when external forces $F=2.5$ and $F=7.0$, and (c) amplitude-frequency response curves when mass parameters $\beta=0.9$ and $\beta=0.7$.

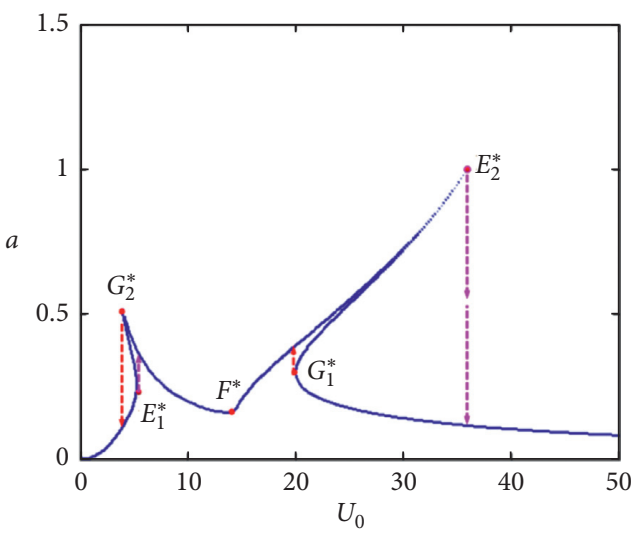

(a)

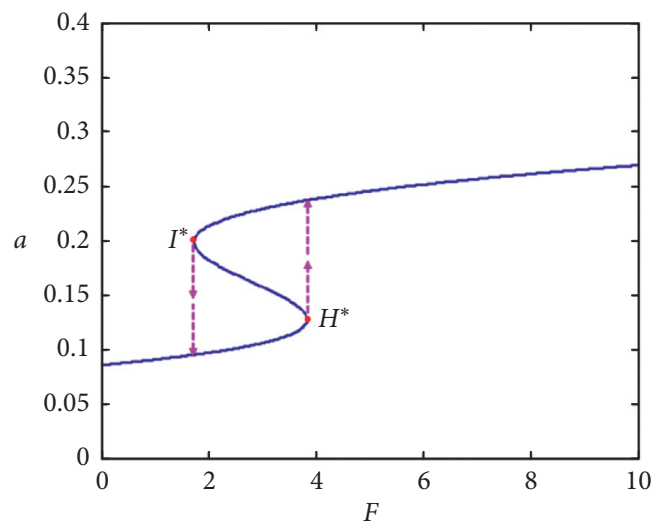

(b)

FIGURE 5: The amplitude-frequency response curves of the cantilevered pipe conveying pulsating fluid are obtained. (a) Amplitude-response curves on flow velocity; (b) amplitude-response curves on external excitation. 

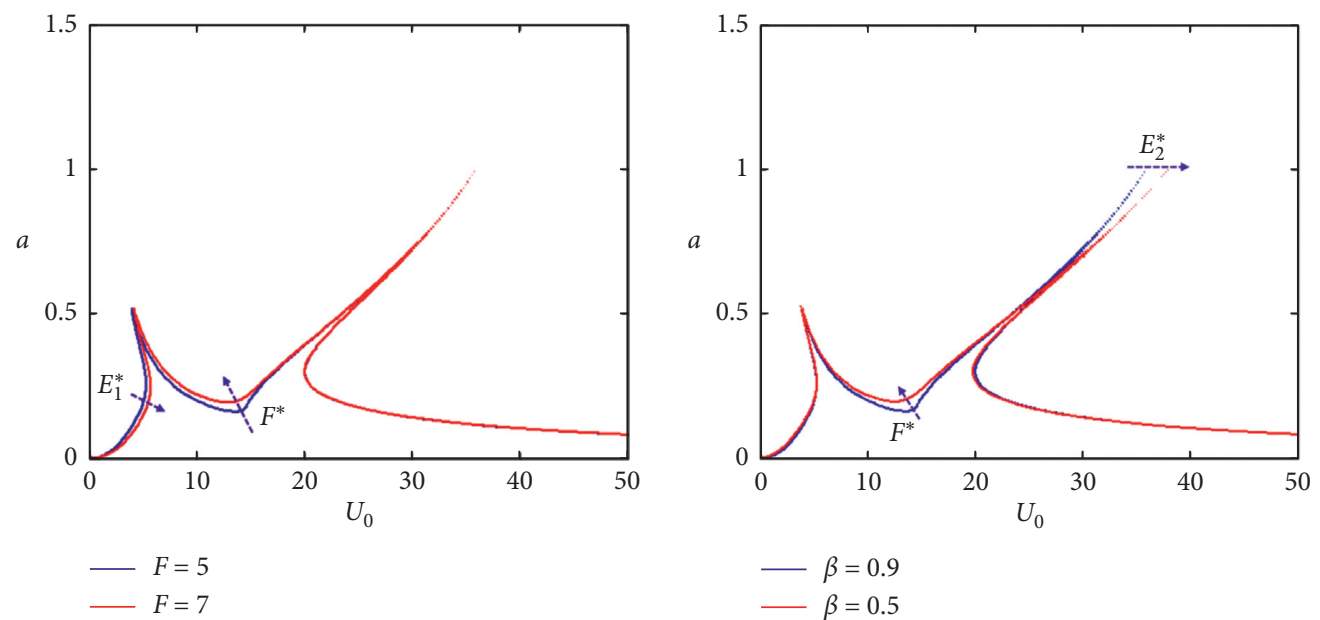

(a)

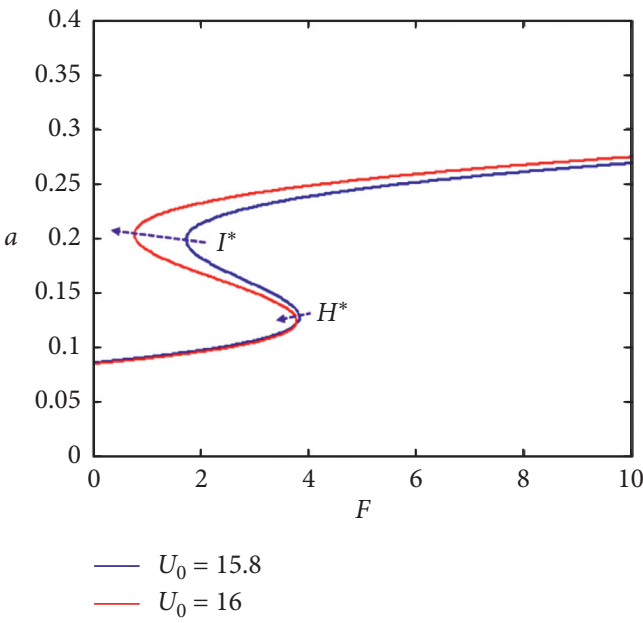

(c)

(b)

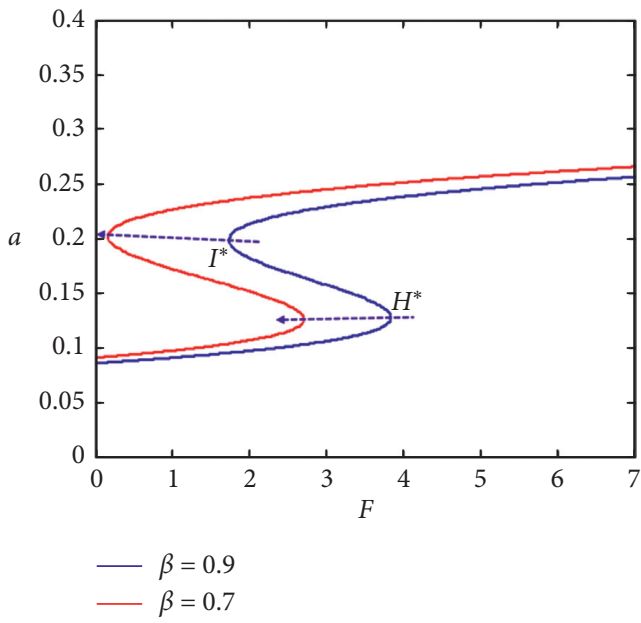

(d)

Figure 6: The influences of different parameters on the amplitude-frequency response curves and force-amplitude response curves are given for the cantilevered pipe conveying pulsating fluid. (a) Amplitude-frequency response curves on flow velocity when $F=5$ and $F=7$, (b) amplitudefrequency response curves on flow velocity when mass parameters $\beta=0.9$ and $\beta=0.5$, (c) force-amplitude response curves on external force when flow velocities $U_{0}=15.8$ and $U_{0}=16$, and (d) force-amplitude response curves on external force when mass parameters $\beta=0.9$ and $\beta=0.7$.

Figures 6(a)-6(d), the changes of the amplitude-frequency and force-frequency response curves in each figure are caused by changing one parameter while other parameters remain unchanged. The influences of different parameters, such as the parametric excitation and mass parameter, on the amplitudefrequency response curves are studied, as shown in Figures 6(a) and 6(b). Figure 6(a) gives the relations between the amplitudes and flow velocity $U_{0}$ for the cantilevered pipe conveying pulsating fluid when the parametric excitations induced to the flow velocity are $F=5.0$ and $F=7.0$, respectively. Figure 6(b) indicates the relations on the amplitudes versus the flow velocity $U_{0}$ in the cantilevered pipe conveying pulsating fluid when the mass parameters are $\beta=0.9$ and $\beta=0.7$, respectively. We find that the amplitudes of the nonlinear oscillations for the cantilevered pipe conveying pulsating fluid become larger as the parametric excitation increases and the mass parameter decreases. This indicates that when the pipe mass is lighter, the amplitudes of the nonlinear oscillations are greater. In Figures 6(c) and 6(d), we study the influences of the external excitations on the force-amplitude response curves. Figure 6(c) demonstrates the relations between the amplitudes versus the external excitations $F$ in the cantilevered pipe conveying pulsating fluid when the flow velocities are $U_{0}=15.8$ and $U_{0}=16.0$, respectively. We find that the amplitudes of the nonlinear oscillations for the cantilevered pipe conveying pulsating fluid become larger when the flow velocity is larger. Figure 6(d) shows the relations on the amplitudes versus the external excitations $F$ in the cantilevered pipe conveying pulsating fluid when the mass parameters are $\beta=0.9$ and $\beta=0.7$, respectively. It is found that the nonlinear oscillations of the cantilevered pipe conveying pulsating fluid more easily happen with the decrease of the mass parameter $\beta$.

Based on the analyses of the amplitude-frequency response curves, it can be found that the hardening-spring type behaviors and jumping phenomena are exhibited for the cantilevered pipe conveying pulsating fluid. The jumping phenomena also occur in the force-amplitude response curves versus the flow velocity and external excitation. Moreover, it is known that the flow 
velocity, external excitation, and coupling degree of two oscillation modes can affect the nonlinear oscillations of the cantilevered pipe conveying pulsating fluid under the external harmonic force. The nonlinearity bends the amplitude-frequency response curves to the right when the cantilevered pipe conveying pulsating fluid has the hardening-spring type behaviors. The bending of the amplitude-frequency response curves leads to the occurrence of the jumping phenomena. The hardening-spring type behavior and jumping phenomena mean that the oscillation amplitudes of the cantilevered pipe conveying pulsating fluid increase firstly and change abruptly. The energy of the fluid motion is transferred into the energy of the cantilevered pipe when the flow velocity increases. Thus, the fluid-structure interaction happens for the cantilevered pipe conveying pulsating fluid. The nonlinear resonance of the large amplitude for the cantilevered pipe conveying pulsating fluid can be stimulated with the increase of these factors.

\section{Numerical Simulations of Periodic and Chaotic Oscillations}

In order to study the nonlinear dynamic properties of the cantilevered pipe conveying pulsating fluid, the influences of the velocity parametric excitation and external excitation on the nonlinear oscillations of the pipe are investigated. In this section, the average equations (23a)-(23d) in the Cartesian coordinate system are numerically simulated to find the multitime periodic and the chaotic oscillations of the cantilevered pipe conveying pulsating fluid under the external harmonic excitation based on the fourth-order Runge-Kutta algorithm [50-52]. We choose the velocity parametric and external excitations as the controlling parameters to study the complicated nonlinear dynamics of the cantilevered pipe conveying pulsating fluid, respectively. The initial conditions are chosen as $x_{1}=0$, $x_{2}=0.1, x_{3}=0$, and $x_{4}=0.1$. Other parameters are chosen as

$$
\begin{aligned}
\beta & =0.9, \\
\gamma & =7, \\
\mu & =15, \\
\sigma & =7.7, \\
\Omega & =15, \\
\Omega_{1} & =8.6, \\
\alpha & =0.7, \\
p_{0} & =-3, \\
p_{1} & =0.5, \\
p_{2} & =-11, \\
p_{3} & =2, \\
p_{4} & =8, \\
p_{5} & =-10, \\
p_{6} & =-13, \\
p_{7} & =16, \\
p_{8} & =-10, \\
p_{9} & =-1,
\end{aligned}
$$

$$
\begin{aligned}
& p_{10}=-10, \\
& p_{11}=-3, \\
& p_{12}=-14, \\
& p_{13}=-6, \\
& p_{14}=-1, \\
& p_{15}=14, \\
& p_{16}=10, \\
& p_{17}=4, \\
& p_{18}=-9, \\
& p_{19}=2, \\
& p_{20}=1, \\
& p_{21}=4, \\
& p_{22}=1, \\
& p_{23}=1, \\
& p_{24}=6, \\
& q_{1}=-5, \\
& q_{2}=-6, \\
& q_{3}=-4.7, \\
& q_{4}=18, \\
& q_{5}=18, \\
& q_{6}=7, \\
& q_{7}=4, \\
& q_{8}=14, \\
& q_{9}=-1, \\
& q_{10}=-11, \\
& q_{11}=9, \\
& q_{12}=-10, \\
& q_{13}=3 .
\end{aligned}
$$

To reveal the nonlinear dynamic behaviors of the cantilevered pipe conveying pulsating fluid, the bifurcation diagrams, maximum Lyapunov exponents, phase portraits, waveforms, and Poincare map are depicted. Figures 7 and 8 present the bifurcation diagrams and maximum Lyapunov exponents to demonstrate the multitime nonlinear oscillation of the cantilevered pipe conveying pulsating fluid by varying the velocity parametric excitation and external excitation, respectively.

In order to study the influences of the flow velocities on the multitime nonlinear oscillations of the cantilevered pipe conveying pulsating fluid, we set the external excitation while other parameters remain unchanged. The bifurcation diagrams and maximum Lyapunov exponents of the 


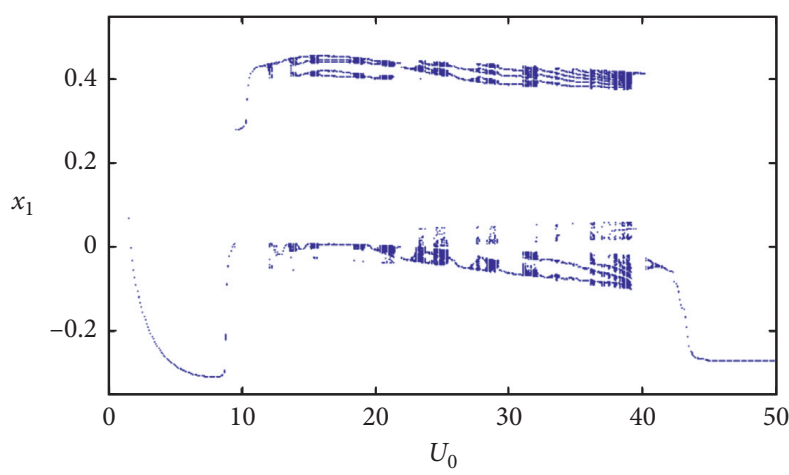

(a)

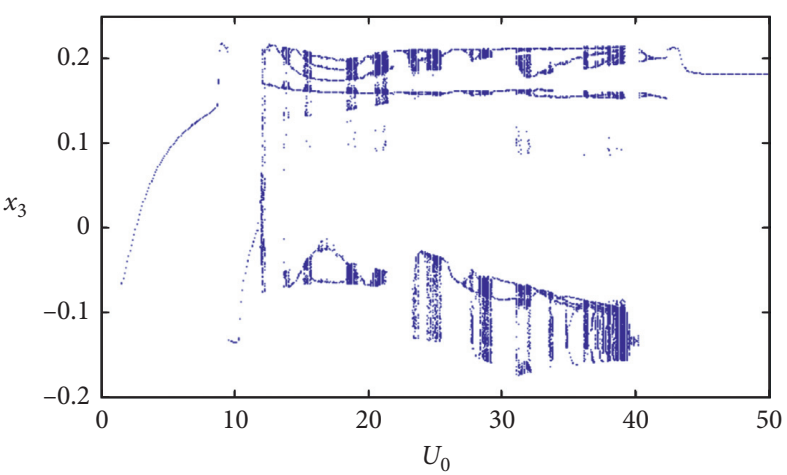

(b)

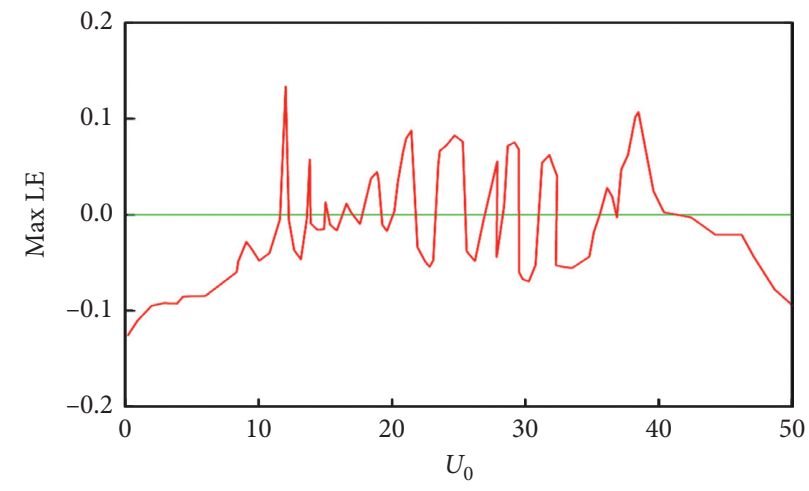

(c)

Figure 7: The bifurcation diagrams and maximum Lyapunov exponents of the cantilevered pipe conveying pulsating fluid are given for the parametric excitations of the flow velocities when the external excitation is $F=7$. (a) $x_{1}$ versus flow velocity, (b) $x_{3}$ versus flow velocity, and (c) maximum Lyapunov exponents versus flow velocity.

cantilevered pipe conveying pulsating fluid with the change of the flow velocity are shown in Figure 7 when the external excitation $F=7$. According to changing characteristics of the bifurcation diagram, with the increase of the flow velocities, the period oscillations of the cantilevered pipe conveying pulsating fluid appear at the beginning, the multitime quasi-period oscillations appear, and the multitime chaotic oscillations happen. With the increase of the flow rate, the multiple periodic windows appear for the cantilevered pipe conveying pulsating fluid. This illustrates the alternating change of the periodic and chaotic oscillations. When the flow rate increases to a particular value, the motion of the cantilevered pipe conveying pulsating fluid evolves to a stable period-1 oscillation. The maximum Lyapunov exponents given in Figure 7(c) describe the intervals of the flow velocity for the chaotic vibrations.

Based on numerical simulations corresponding to the aforementioned analyses, we further investigate the effects of the external excitations on the nonlinear dynamics of the cantilevered pipe conveying pulsating fluid when the flow velocity is $U_{0}=12$ and other parameters remain unchanged, as shown in Figure 8. It is found that the changing characteristics of the oscillations with the increase of the external excitations are similar to those with the increase of the flow velocities for the cantilevered pipe conveying pulsating fluid. The periodic oscillation appears at the beginning, and then the multitime periodic oscillation appears, and, finally, the multitime chaotic oscillations occur for the cantilevered pipe conveying pulsating fluid under the external harmonic excitation. With the further increase of the external excitation, several periodic oscillation windows appear for the cantilevered pipe conveying pulsating fluid. When the external excitation increases to a particular value, the motions of the cantilevered pipe conveying pulsating fluid eventually become the chaotic oscillations from the intermittent chaos. The intermittent chaos is verified in Figure 8(c) by whether the maximum Lyapunov exponent is positive.

We present a variety of figures to confirm the vibrations of the cantilevered pipe conveying pulsating fluid corresponding to different flow rates and different external excitations. In Figures 9-17, Figures(a) and (c) represent the phase portraits on the planes $\left(x_{1}, x_{2}\right)$ and $\left(x_{3}, x_{4}\right)$, Figures (b) and (d) depict the waveforms on the planes $\left(t, x_{1}\right)$ and $\left(t, x_{3}\right)$, Figure(e) denotes three-dimensional phase portrait in the space $\left(x_{1}, x_{2}, x_{3}\right)$, and Figure $(g)$ depicts Poincare map on the plane $\left(x_{1}, x_{2}\right)$, respectively. The aforementioned 


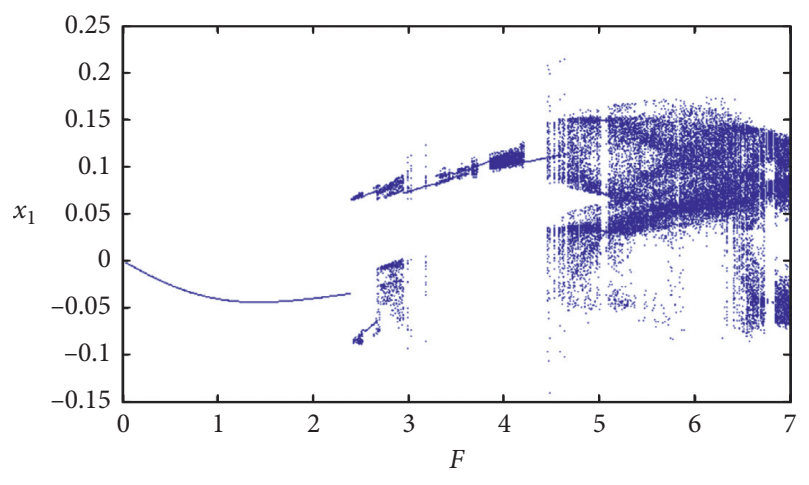

(a)

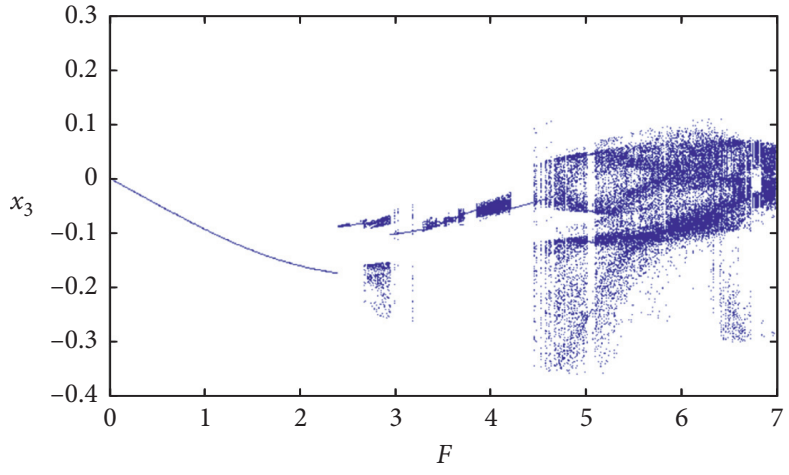

(b)

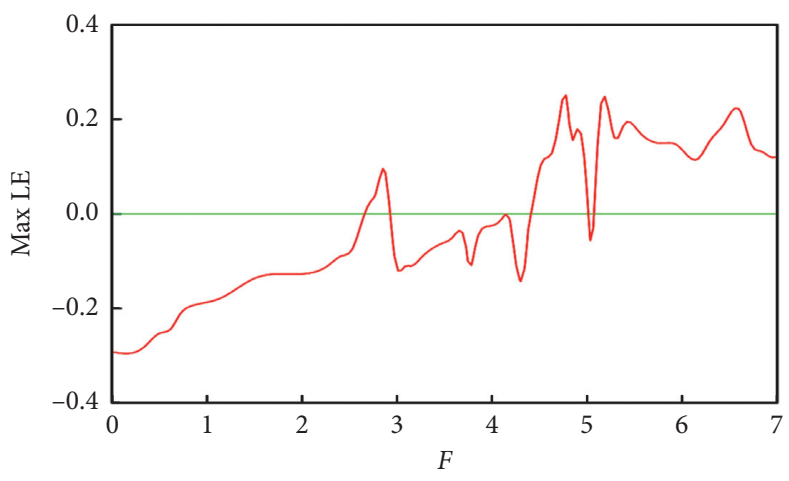

(c)

Figure 8: The bifurcation diagrams and maximum Lyapunov exponents of the cantilevered pipe conveying pulsating fluid are obtained for the external excitations when the flow velocity is $U_{0}=12$. (a) $x_{1}$ versus external excitation, (b) $x_{3}$ versus external excitation, and (c) maximum Lyapunov exponents versus flow velocity.

figures are the effective tools to judge the chaotic oscillations of the cantilevered pipe conveying pulsating fluid. Whether the waveforms are regular or irregular, these indicate that the motions of the cantilevered pipe conveying pulsating fluid are the periodic or chaotic oscillations. The phase diagram is the projection curve of the solution in the phase plane and phase space. The closed curve represents the periodic oscillation, and the phase portraits of the chaotic oscillations are the repeating unclosed curve confined within a bounded region. According to Poincare mapping, the periodic and quasi-periodic oscillations are the isolated points and curves on Poincare cross section. The nonlinear oscillations of the cantilevered pipe conveying pulsating fluid are the multitime chaotic oscillations when the discrete point sets appear with the infinite self-similar structures on Poincare cross section.

According to the characteristics of each figure for different motions, there exist the periodic, multitime quasiperiodic, and multitime chaotic oscillations of the cantilevered pipe conveying pulsating fluid under different velocity parametric excitations, as shown in Figures 9-13. Figure 9 gives the periodic oscillation of the cantilevered pipe conveying pulsating fluid when the velocity parametric excitation is $U_{0}=11.0$. Figure 10 indicates that there exists the period-2 oscillation of the cantilevered pipe conveying pulsating fluid when the velocity parametric excitation is $U_{0}=19.5$. Based on the definition of the coordinate system, we know that the right is positive and the left is negative for the cantilevered pipe conveying pulsating fluid. It is found that, in the cantilevered pipe conveying pulsating fluid, there are the nonlinear oscillations around the left-mode and right-mode positions, respectively. The first-order and thirdorder oscillation modes of the cantilevered pipe conveying pulsating fluid vibrate twice times around the right-mode position and then vibrate twice times around the left-mode position, as shown in Figures 10(b) and 10(d). Between the left-mode and right-mode nonlinear oscillations, there exists one-time oscillation around the vertical equilibrium position in the cantilevered pipe conveying pulsating fluid. This is a typical nonlinear dynamic phenomenon.

Figure 11 illustrates that the multiperiodic oscillation happens when the velocity parametric excitation is $U_{0}=23.2$. From Figure 11(b), it is demonstrated that, in the first-order mode of the nonlinear oscillations, there exists one-time oscillation around the right-mode position and then there exists twice-time oscillation around the left-mode position. For the third-order mode, there exists twice-time 


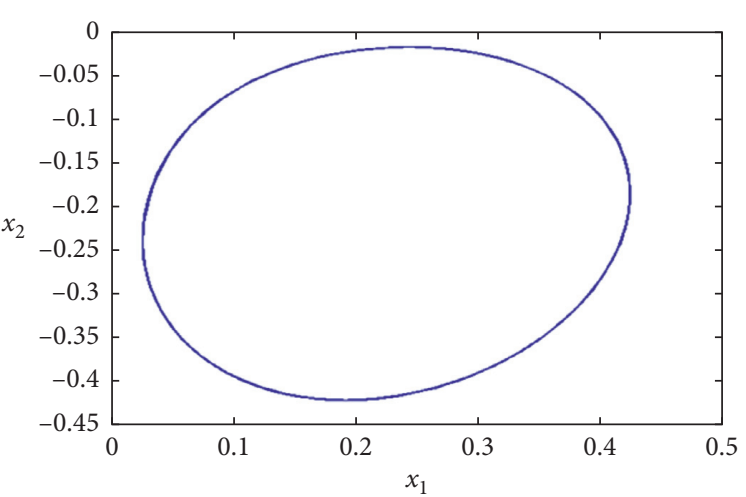

(a)

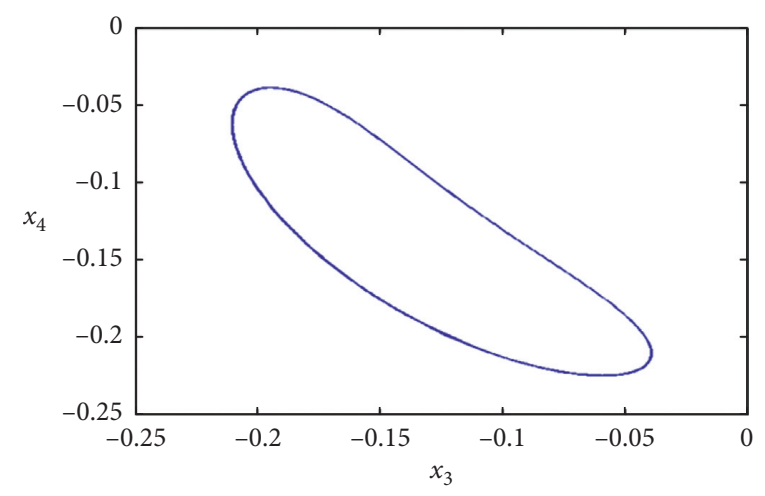

(c)

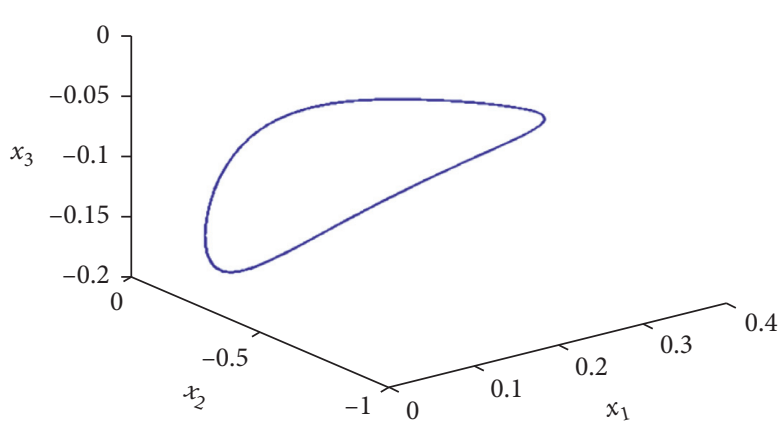

(e)

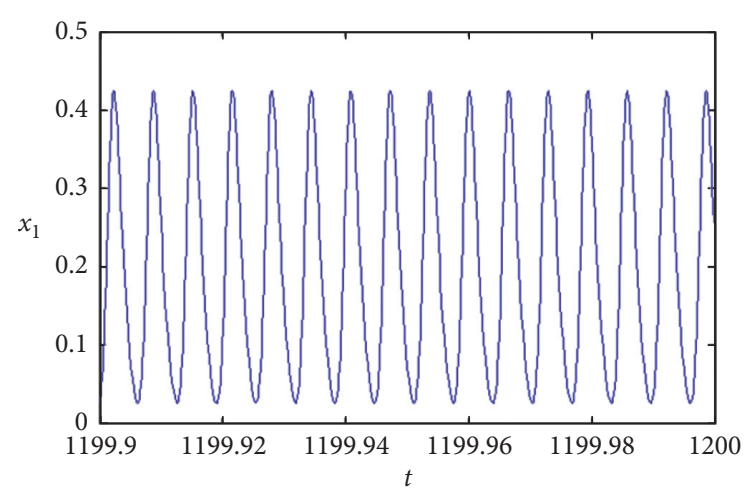

(b)

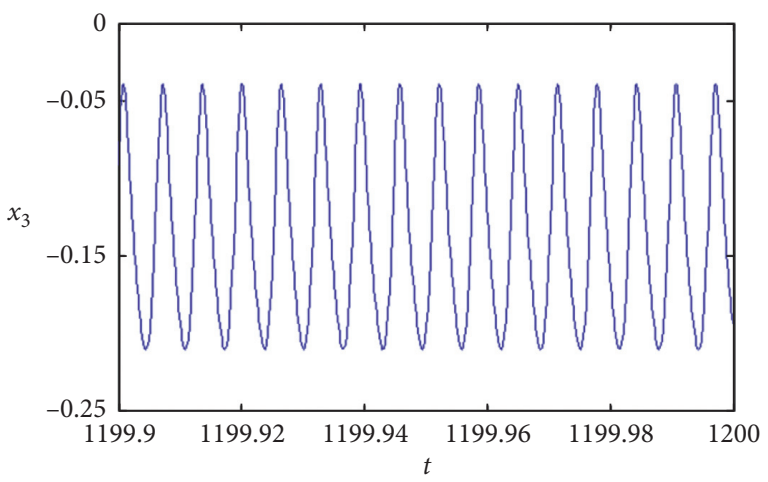

(d)

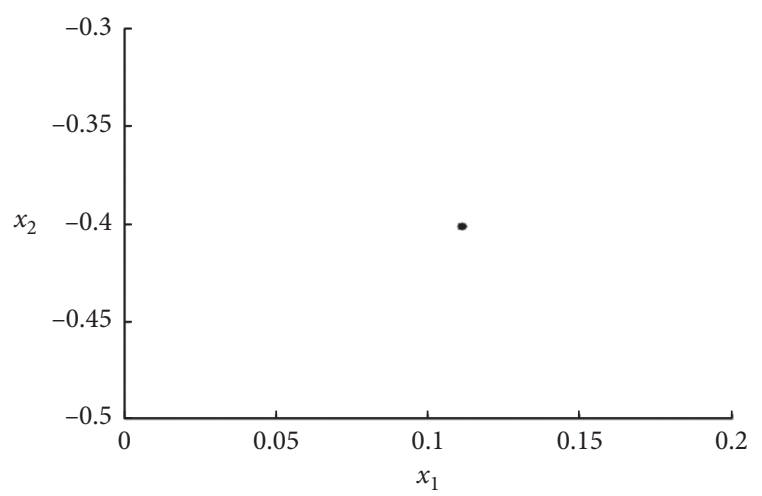

(f)

FIgURE 9: The periodic oscillation of the cantilevered pipe conveying pulsating fluid is obtained when the parametric excitation of the flow velocity is $U_{0}=11.0$. (a) Phase portrait on plane $\left(x_{1}, x_{2}\right)$, (b) waveform on plane $\left(t, x_{1}\right)$, (c) phase portrait on plane $\left(x_{3}, x_{4}\right)$, (d) waveform on plane $\left(t, x_{3}\right)$, (e) three-dimensional phase portrait in space $\left(x_{1}, x_{2}, x_{3}\right)$, and (g) Poincare map on plane $\left(x_{1}, x_{2}\right)$.

oscillation around the right-mode position and then there exists one-time oscillation around the left-mode position in the cantilevered pipe conveying pulsating fluid, as shown in Figure 11(d).

Figure 12 indicates that there exist the multitime chaotic oscillations when the velocity parametric excitation is $U_{0}=31.5$. Around the left-mode and right-mode positions, the chaotic oscillations of the cantilevered pipe conveying pulsating fluid are the irregular motions. Figure 13 indicates that the motion of the cantilevered pipe conveying pulsating fluid again returns to the period-1 oscillation when the velocity parametric excitation is $U_{0}=42.5$. In this case, it is found from Figure 13(b) that the first-order mode of the cantilevered pipe conveying pulsating fluid is the oscillation around the left-mode position. However, the third-order vibration mode of the cantilevered pipe conveying pulsating 


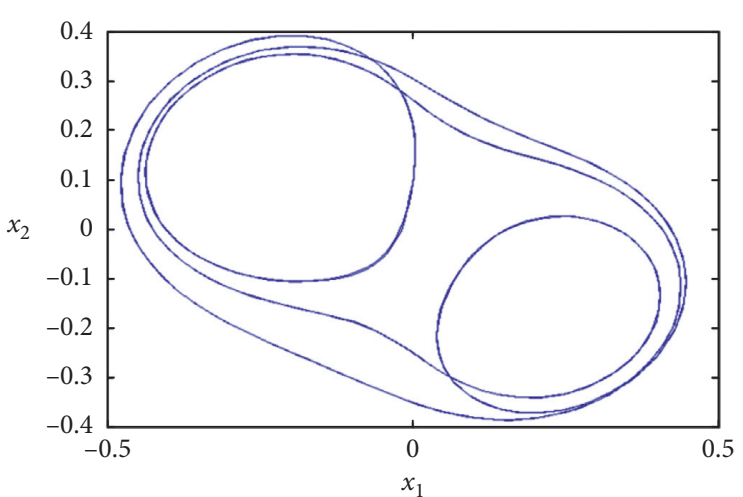

(a)

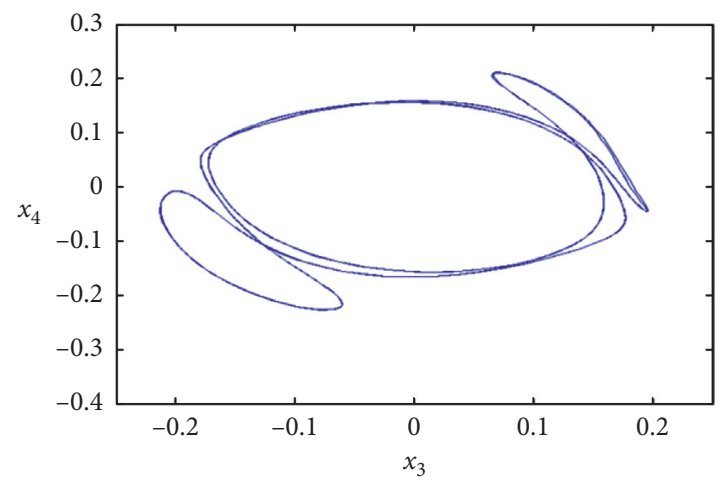

(c)

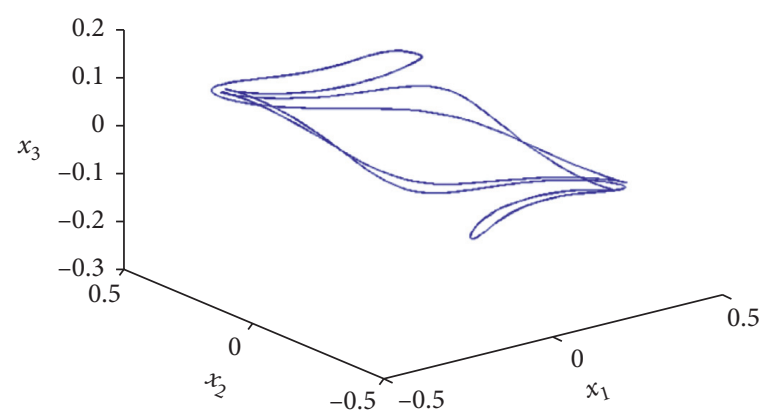

(e)

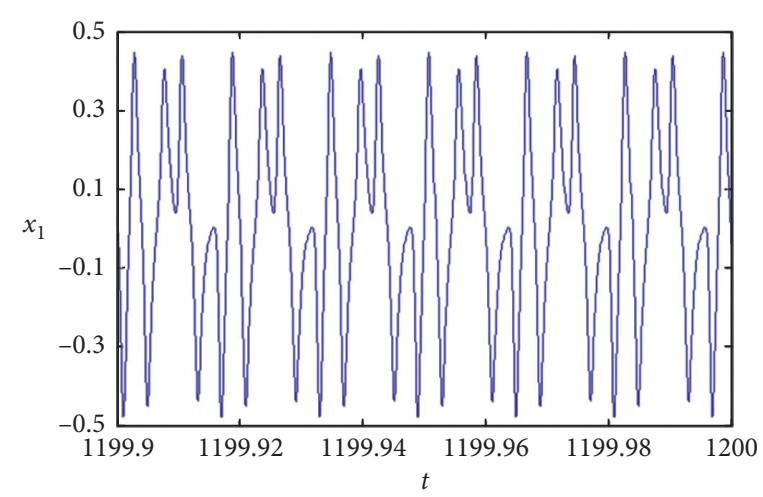

(b)

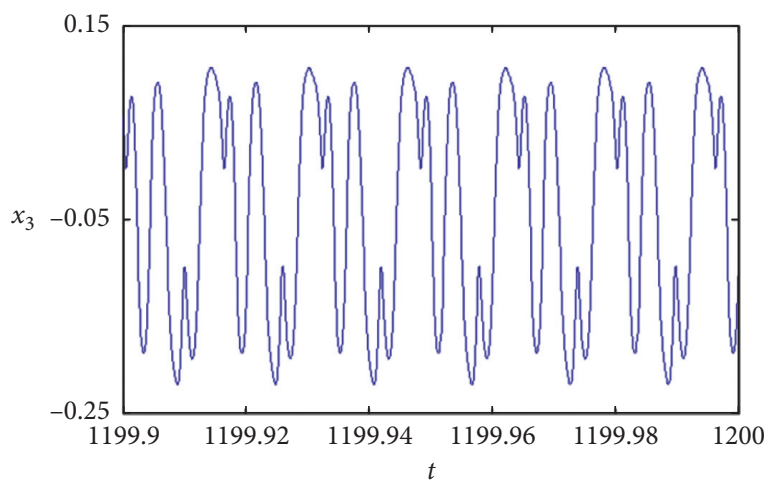

(d)

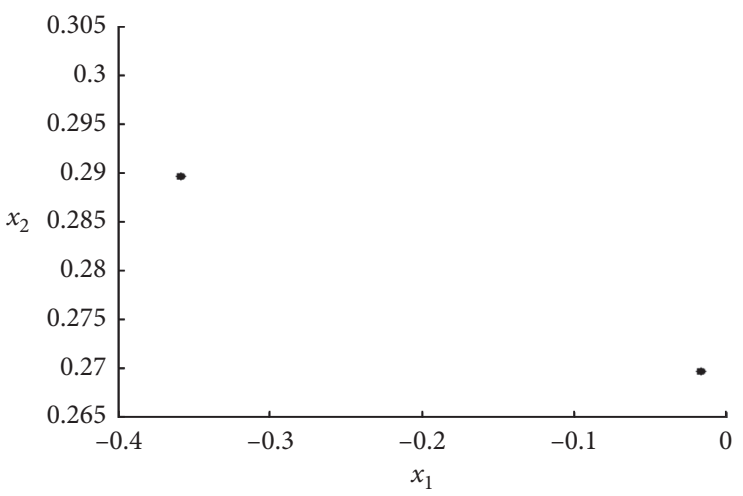

(f)

FIGURE 10: The period-2 oscillation of the cantilevered pipe conveying pulsating fluid is obtained when the parametric excitation of the flow velocity is $U_{0}=19.5$. (a) phase portrait on plane $\left(x_{1}, x_{2}\right)$, (b) waveform on plane $\left(t, x_{1}\right)$, (c) phase portrait on plane $\left(x_{3}, x_{4}\right)$, (d) waveform on plane $\left(t, x_{3}\right)$, (e) three-dimensional phase portrait in space $\left(x_{1}, x_{2}, x_{3}\right)$, and (g) Poincare map on plane $\left(x_{1}, x_{2}\right)$.

fluid is a motion around the right-mode position, as shown in Figure 13(d).

We also find the periodic, multitime periodic, and multitime chaotic oscillations of the cantilevered pipe conveying pulsating fluid under different external excitations, as shown in Figures 14-17. Figure 14 gives the multiperiodic oscillation of the cantilevered pipe conveying pulsating fluid when the external excitation is $F=2$.9. It is observed from Figure 14(b) that, for the first-order mode, the motions of the cantilevered pipe conveying pulsating fluid vibrate three times around the right-mode position and twice times around the left-mode position. For the thirdorder mode, the vibrations of the cantilevered pipe conveying pulsating fluid move twice times around the rightmode position and three times around the left-mode position, as shown in Figure 14(d). In addition, it is observed 


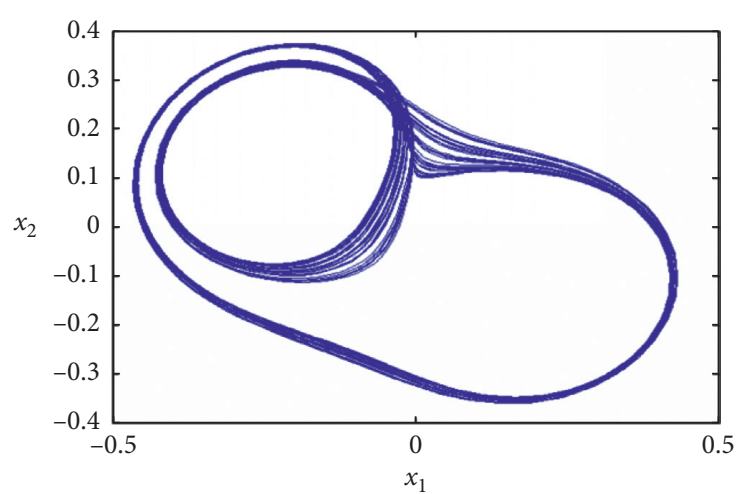

(a)

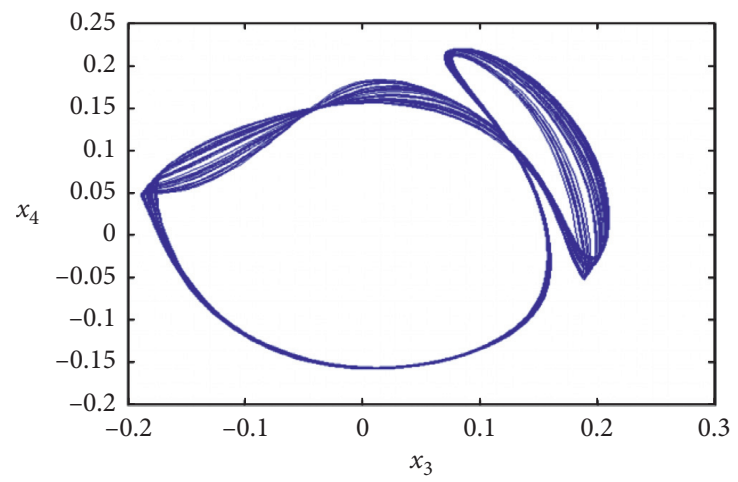

(c)

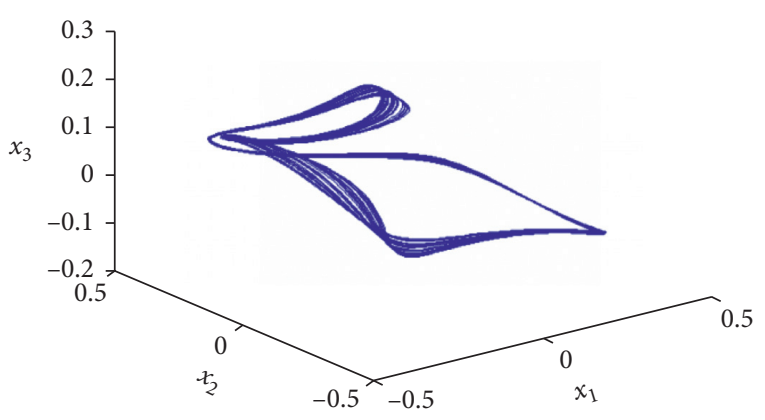

(e)

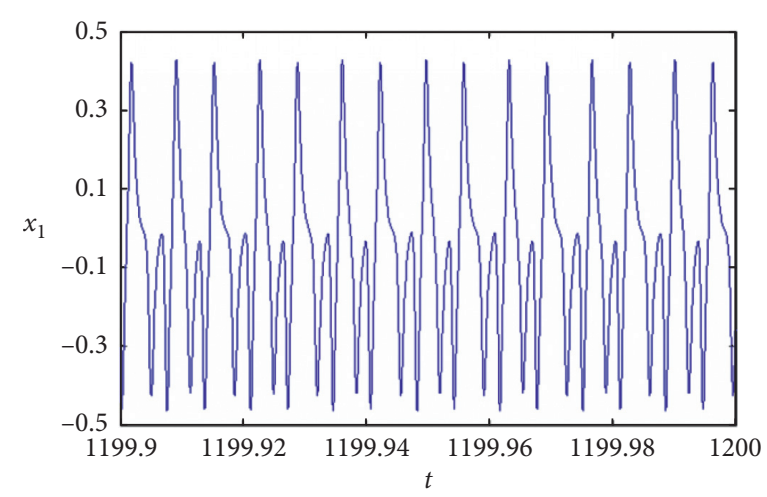

(b)

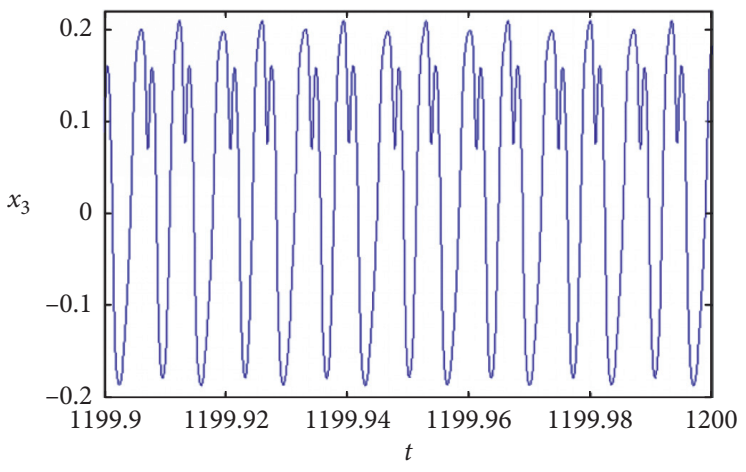

(d)

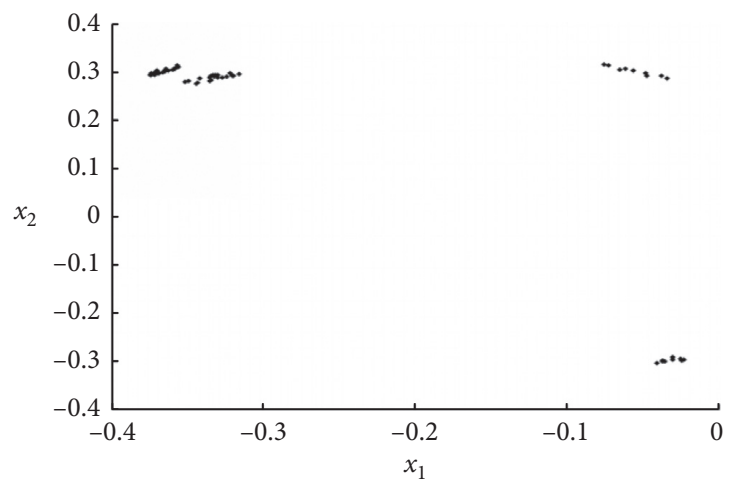

(f)

Figure 11: The multiperiodic oscillation of the cantilevered pipe conveying pulsating fluid is obtained when the parametric excitation of the flow velocity is $U_{0}=23.2$. (a) Phase portrait on plane $\left(x_{1}, x_{2}\right)$, (b) waveform on plane $\left(t, x_{1}\right)$, (c) phase portrait on plane $\left(x_{3}, x_{4}\right)$, (d) waveform on plane $\left(t, x_{3}\right)$, (e) three-dimensional phase portrait in space $\left(x_{1}, x_{2}, x_{3}\right)$, and (g) Poincare map on plane $\left(x_{1}, x_{2}\right)$.

from Figure 14 that the nonlinear oscillations of the cantilevered pipe conveying pulsating fluid also have the motions around the vertical equilibrium position.

Figure 15 indicates that the multitime periodic oscillation happens for the cantilevered pipe conveying pulsating fluid when the external excitation is $F=4.5$. For the firstorder mode, four-time nonlinear oscillations occur around the right-mode position and twice-time nonlinear oscillations occur around the left-mode position in the cantilevered pipe conveying pulsating fluid, as shown in Figure 15(b). It is also found from Figure 15(d) that, for the third-order mode, there are twice-time nonlinear oscillations around the rightmode position and four-time nonlinear oscillations around the left-mode position. Figure 16 represents that the quasiperiodic oscillation occurs when the external excitation is $F=6.3$. Figure 17 demonstrates that there exist the multitime chaotic oscillations of the cantilevered pipe conveying pulsating fluid when the external excitation is $F=6.5$. The 


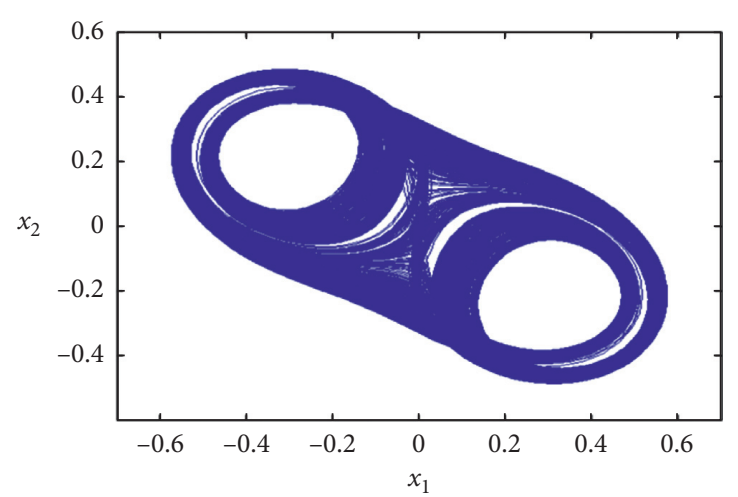

(a)

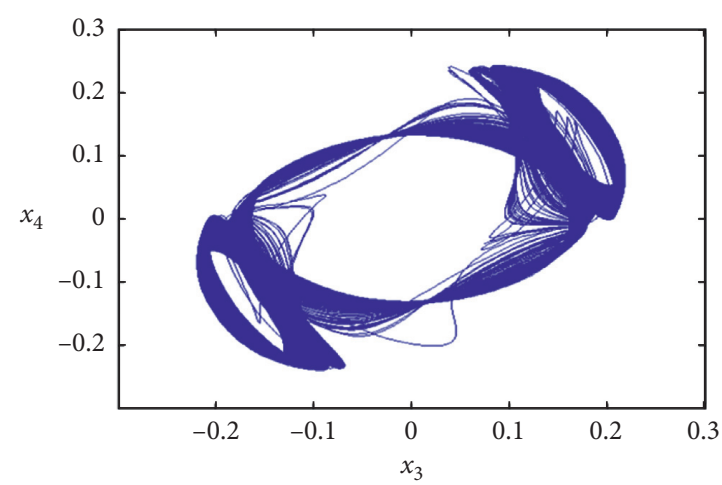

(c)

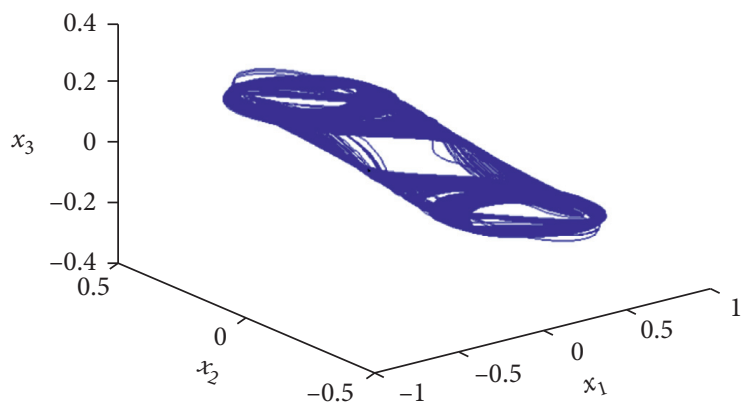

(e)

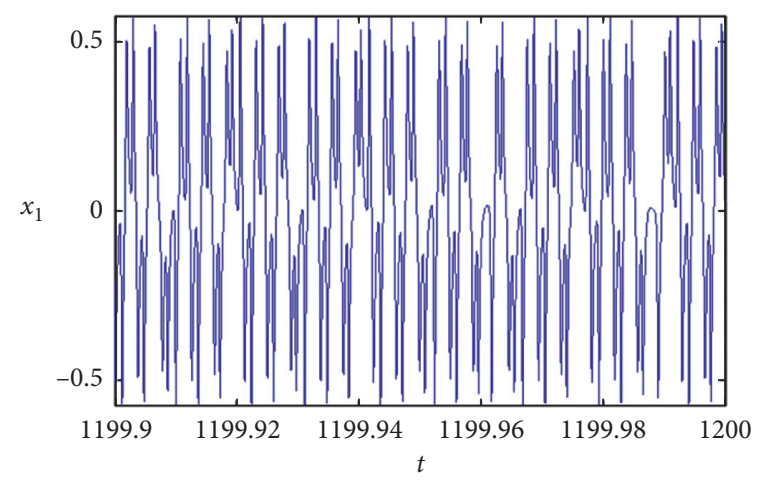

(b)

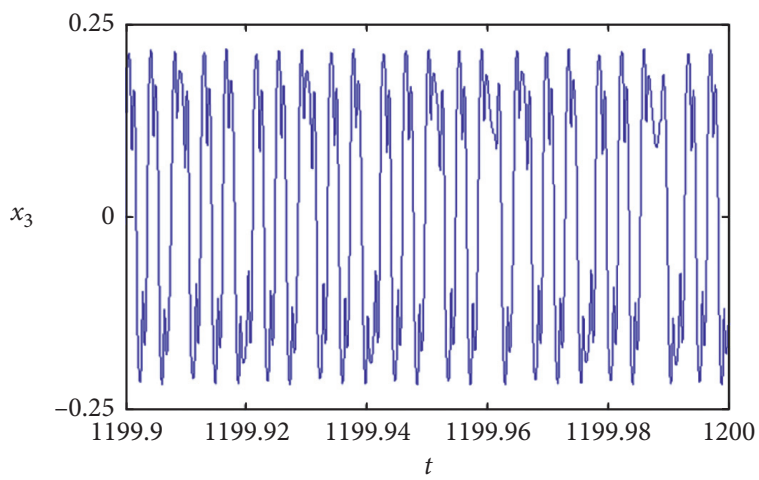

(d)

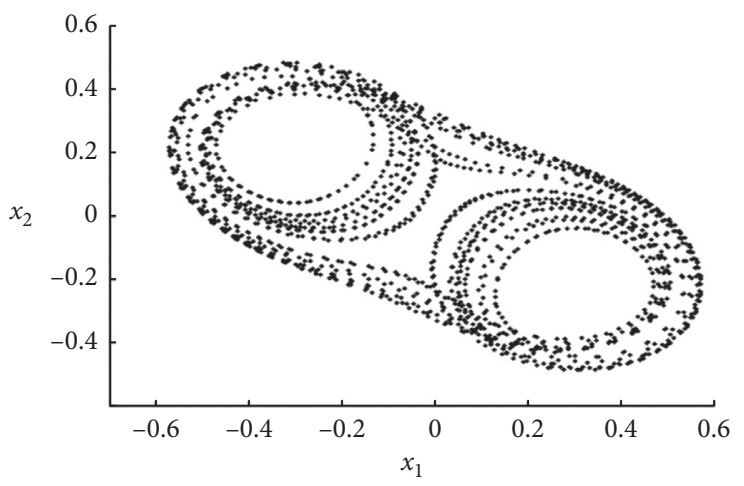

(f)

FIGURE 12: The chaotic oscillations of the cantilevered pipe conveying pulsating fluid are obtained when the parametric excitation of the flow velocity is $U_{0}=31.5$. (a) Phase portrait on plane $\left(x_{1}, x_{2}\right)$, (b) waveform on plane $\left(t, x_{1}\right)$, (c) phase portrait on plane $\left(x_{3}, x_{4}\right)$, (d) waveform on plane $\left(t, x_{3}\right)$, (e) three-dimensional phase portrait in space $\left(x_{1}, x_{2}, x_{3}\right)$, and (g) Poincare map on plane $\left(x_{1}, x_{2}\right)$.

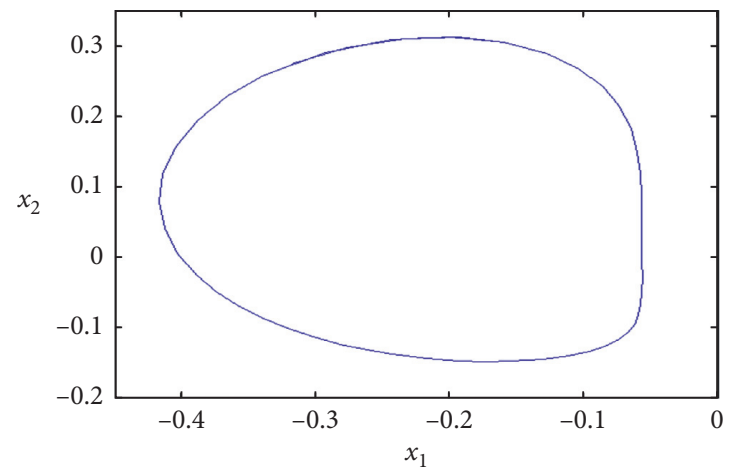

(a)

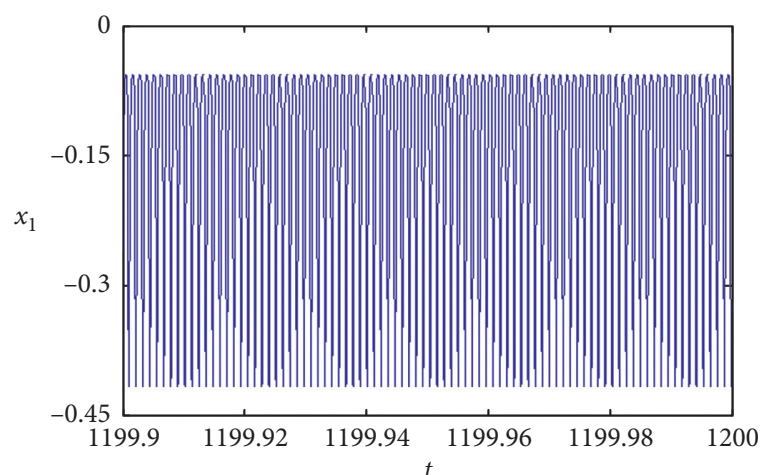

(b)

Figure 13: Continued. 


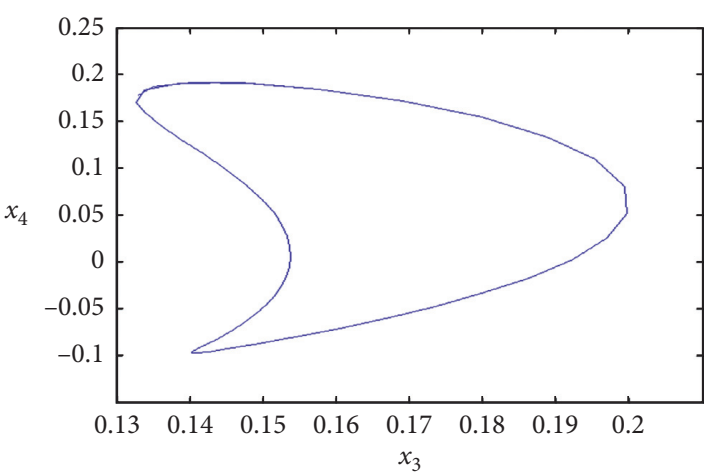

(c)

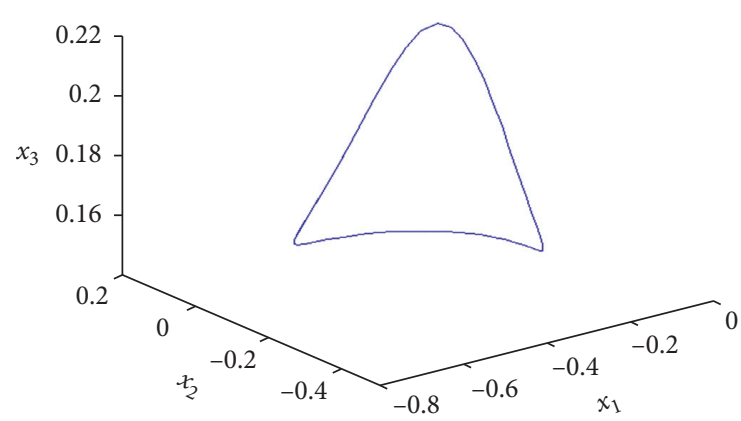

(e)

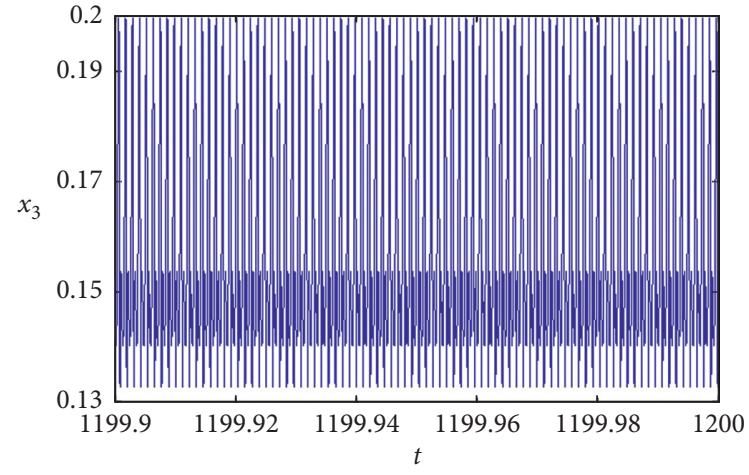

(d)

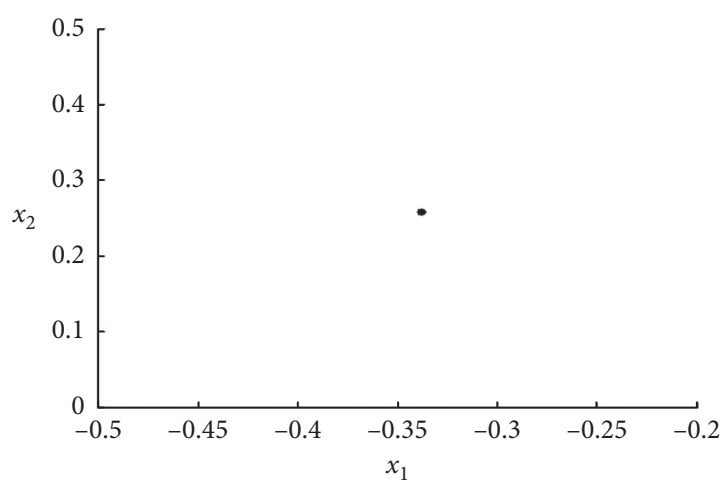

(f)

FIgURE 13: The period-1 oscillation of the cantilevered pipe conveying pulsating fluid is obtained when the parametric excitation of the flow velocity is $U_{0}=42.5$. (a) Phase portrait on plane $\left(x_{1}, x_{2}\right)$, (b) waveform on plane $\left(t, x_{1}\right)$, (c) phase portrait on plane $\left(x_{3}, x_{4}\right)$, (d) waveform on plane $\left(t, x_{3}\right)$, (e) three-dimensional phase portrait in space $\left(x_{1}, x_{2}, x_{3}\right)$, and (g) Poincare map on plane $\left(x_{1}, x_{2}\right)$.

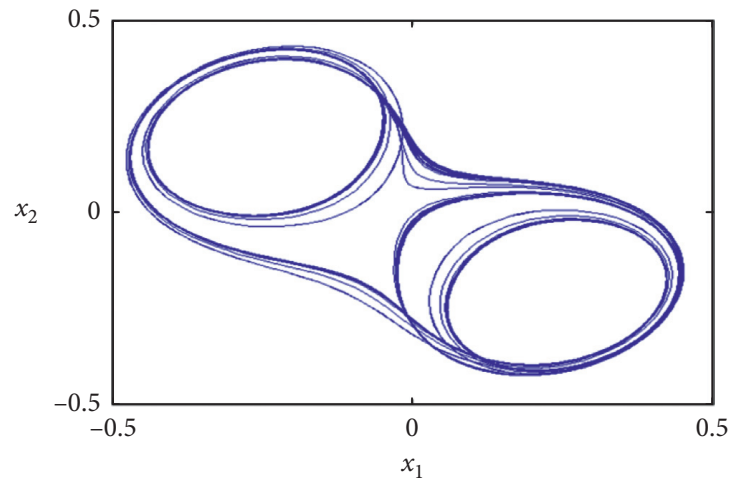

(a)

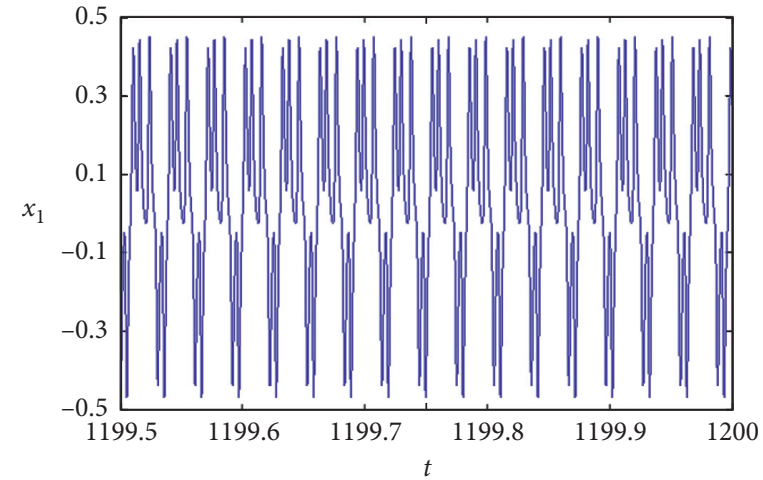

(b)

FIgURE 14: Continued. 


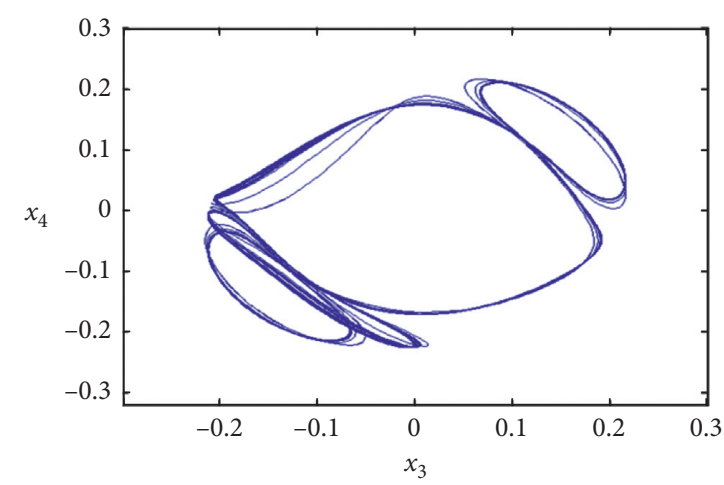

(c)

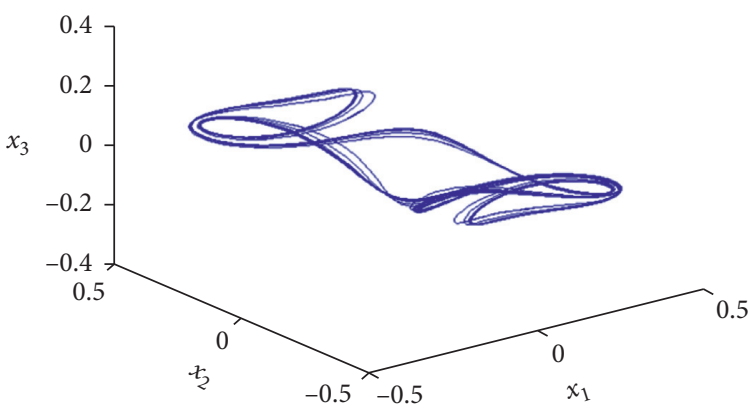

(e)

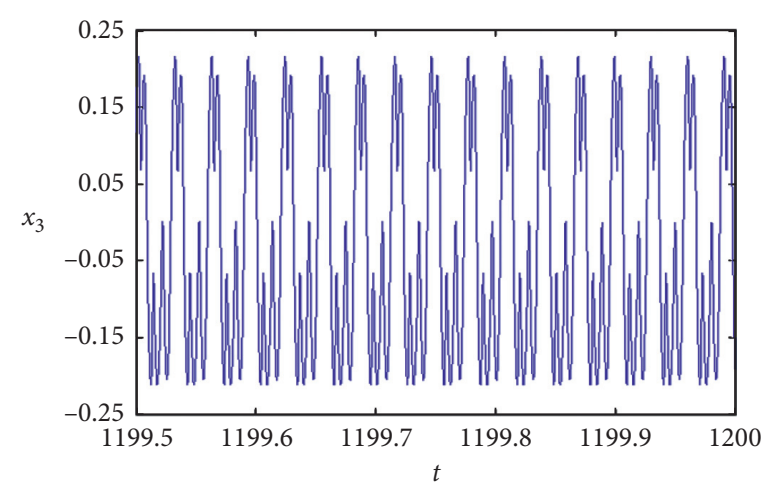

(d)

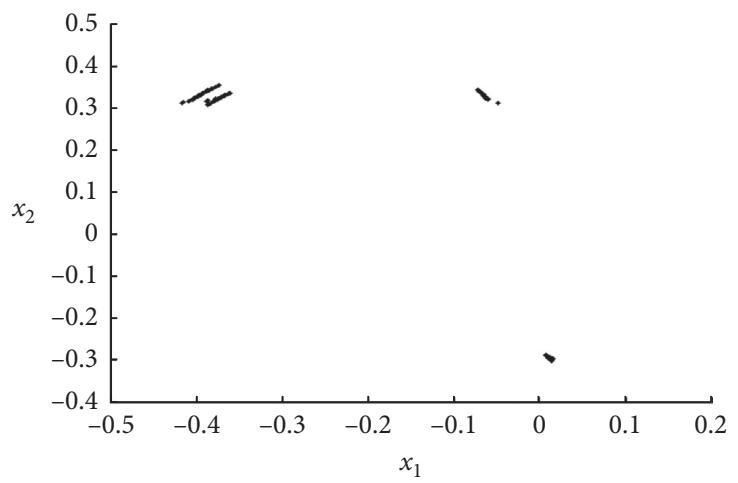

(f)

Figure 14: The multiperiodic oscillation of the cantilevered pipe conveying pulsating fluid is obtained when the external excitation is $F=2.9$. (a) Phase portrait on plane $\left(x_{1}, x_{2}\right)$, (b) waveform on plane $\left(t, x_{1}\right)$, (c) phase portrait on plane $\left(x_{3}, x_{4}\right)$, (d) waveform on plane $\left(t, x_{3}\right)$, (e) three-dimensional phase portrait in space $\left(x_{1}, x_{2}, x_{3}\right)$, and (g) Poincare map on plane $\left(x_{1}, x_{2}\right)$.

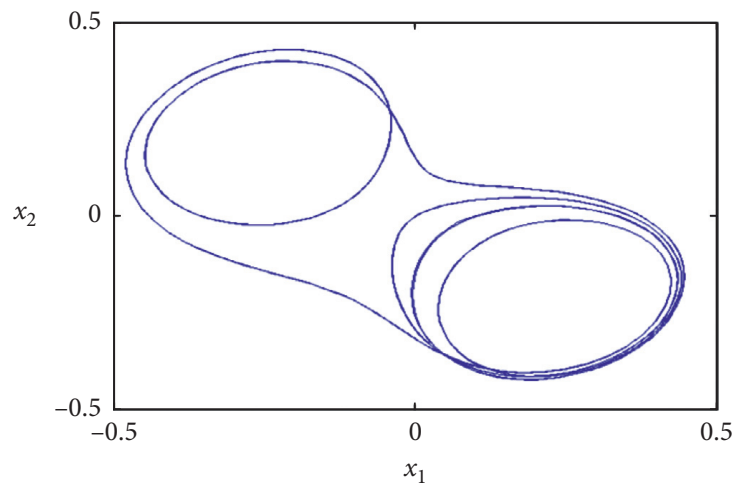

(a)

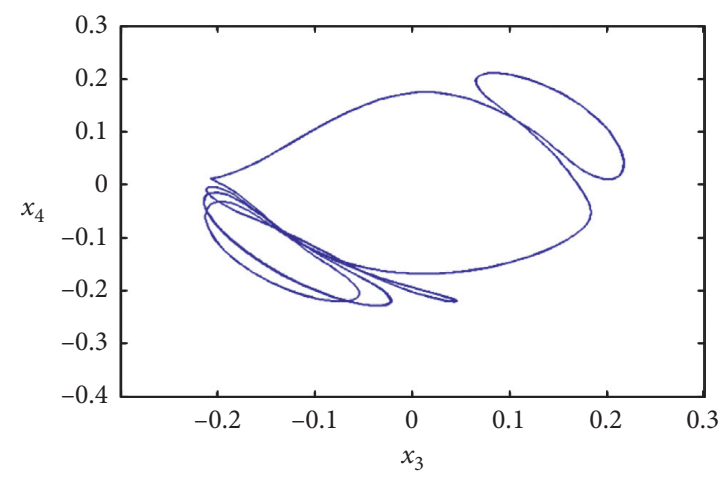

(c)

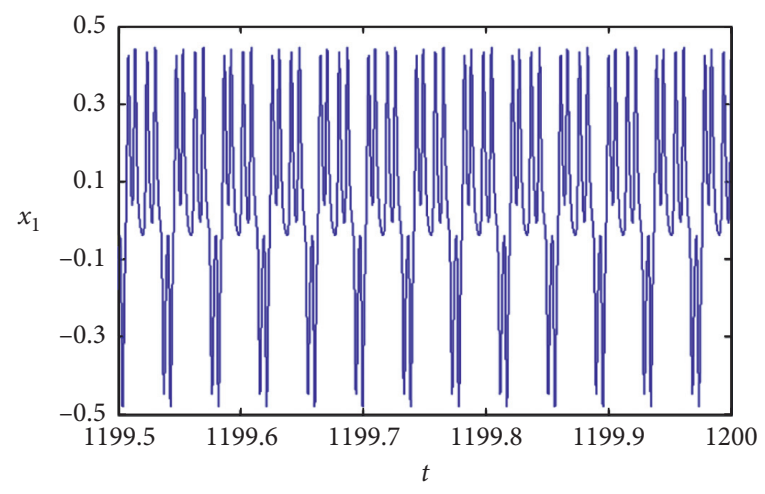

(b)

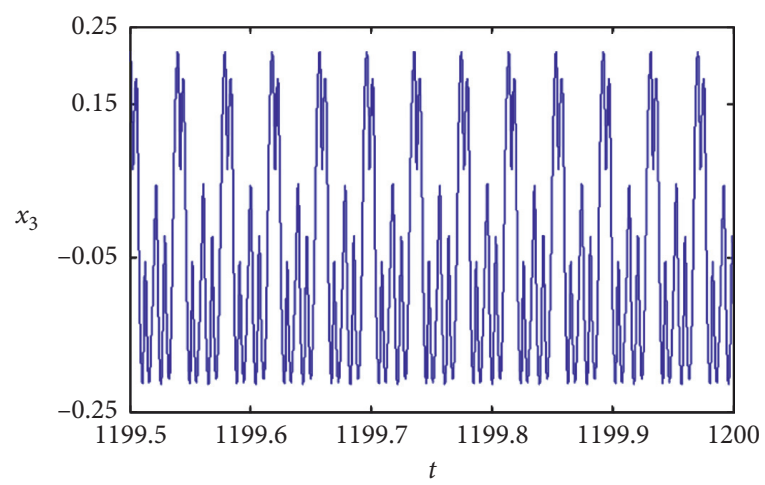

(d)

Figure 15: Continued. 


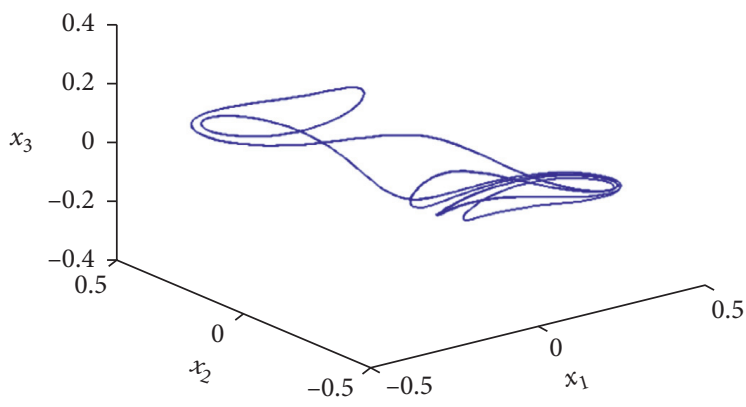

(e)

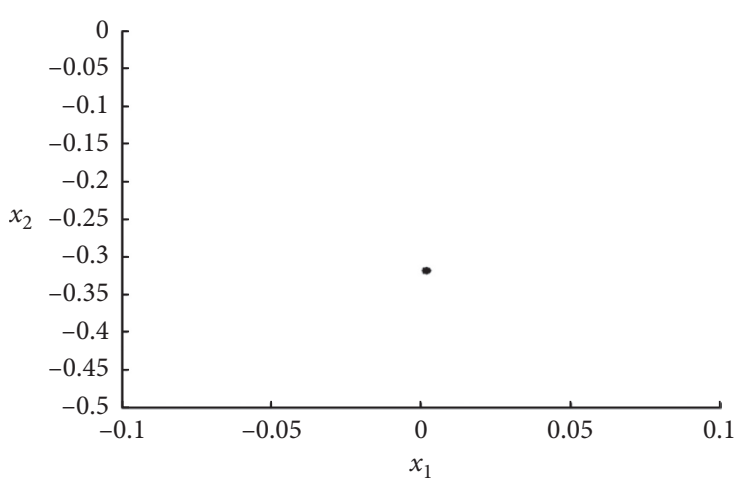

(f)

FIGURE 15: The multitime periodic oscillation of the cantilevered pipe conveying pulsating fluid is obtained when the external excitation is $F=4.5$. (a) Phase portrait on plane $\left(x_{1}, x_{2}\right)$, (b) waveform on plane $\left(t, x_{1}\right)$, (c) phase portrait on plane $\left(x_{3}, x_{4}\right)$, (d) waveform on plane $\left(t, x_{3}\right)$, (e) three-dimensional phase portrait in space $\left(x_{1}, x_{2}, x_{3}\right)$, and (g) Poincare map on plane $\left(x_{1}, x_{2}\right)$.

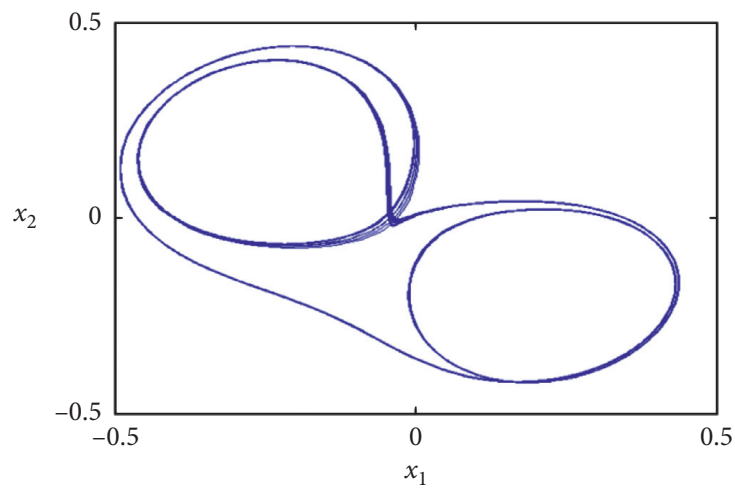

(a)

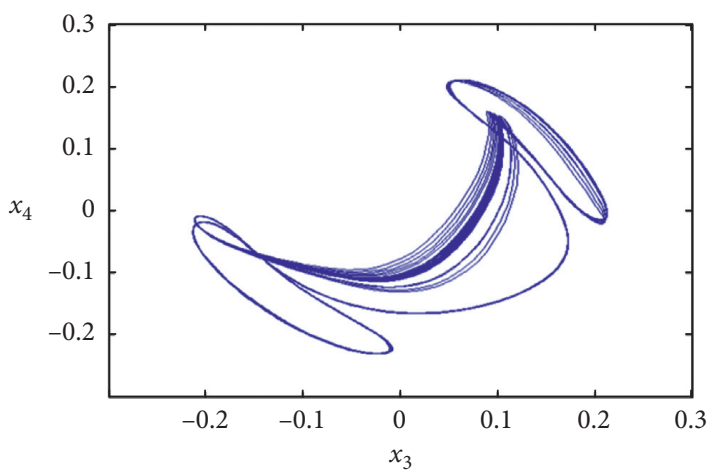

(c)

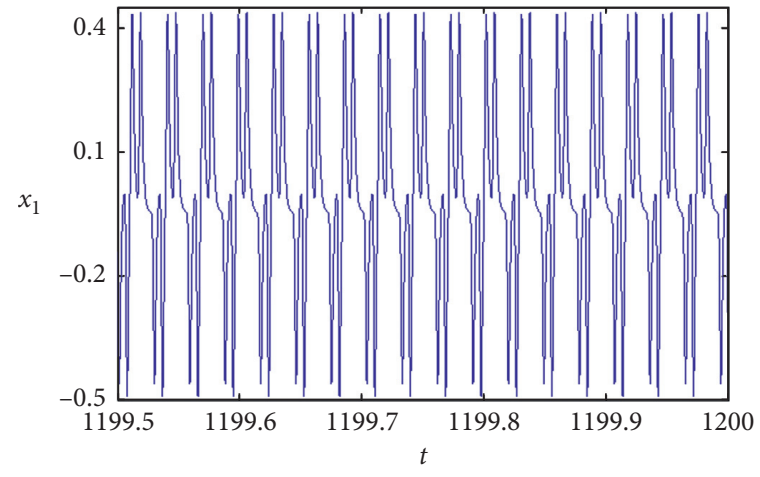

(b)

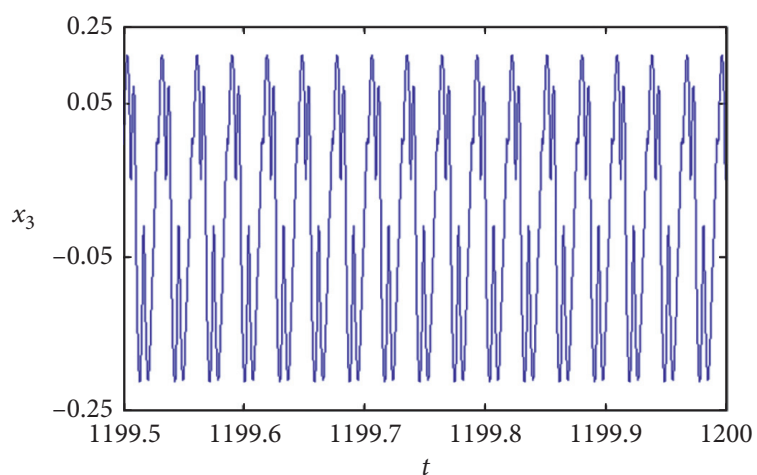

(d)

Figure 16: Continued. 


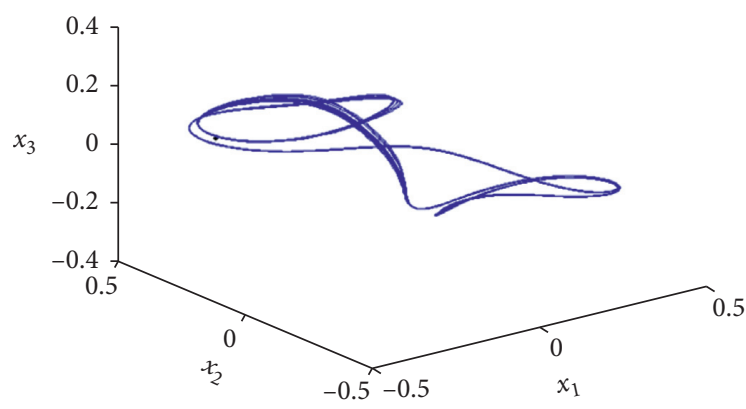

(e)

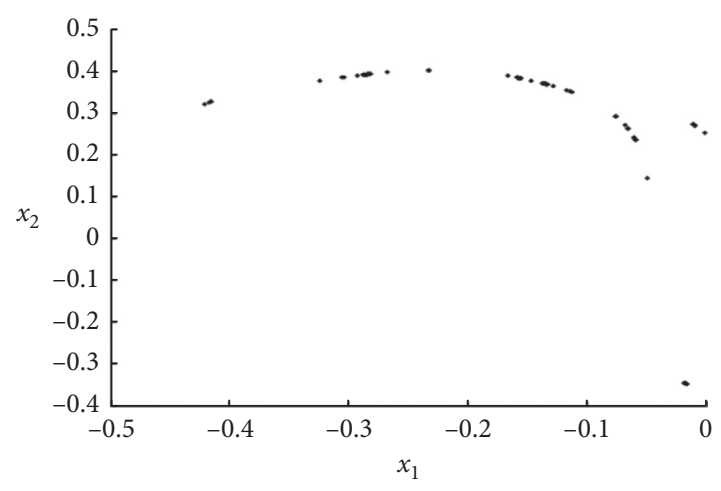

(f)

FIgURE 16: The quasi-periodic oscillation of the cantilevered pipe conveying pulsating fluid is obtained when the external excitation is $F=6.3$. (a) Phase portrait on plane $\left(x_{1}, x_{2}\right)$, (b) waveform on plane $\left(t, x_{1}\right)$, (c) phase portrait on plane $\left(x_{3}, x_{4}\right)$, (d) waveform on plane $\left(t, x_{3}\right)$, (e) three-dimensional phase portrait in space $\left(x_{1}, x_{2}, x_{3}\right)$, and (g) Poincare map on plane $\left(x_{1}, x_{2}\right)$.

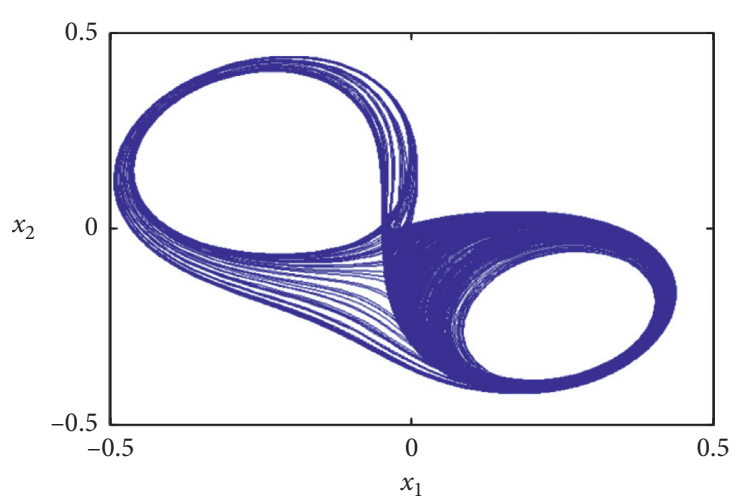

(a)

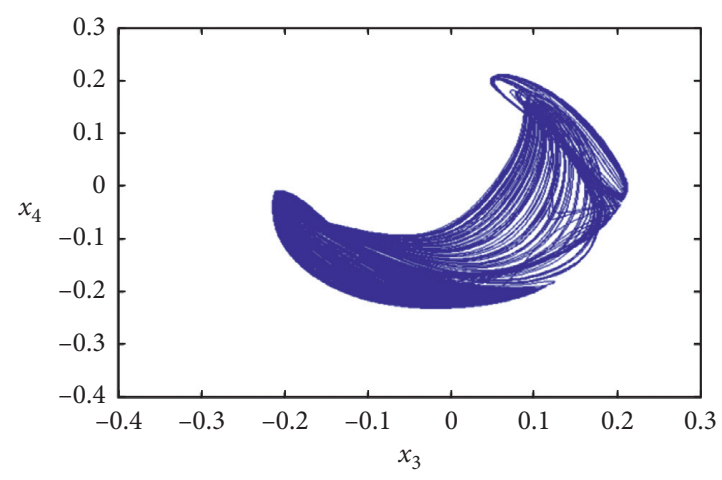

(c)

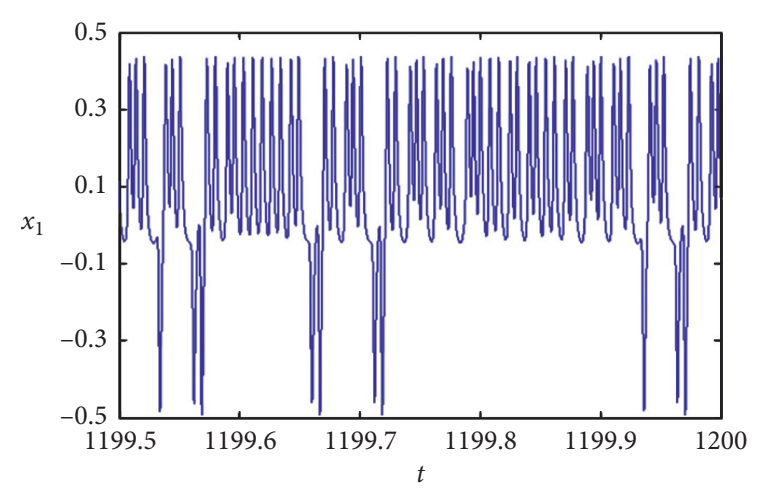

(b)

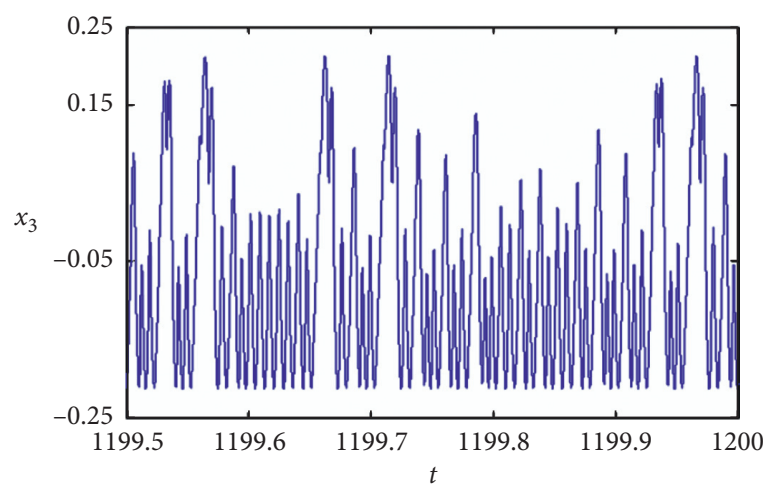

(d)

Figure 17: Continued. 


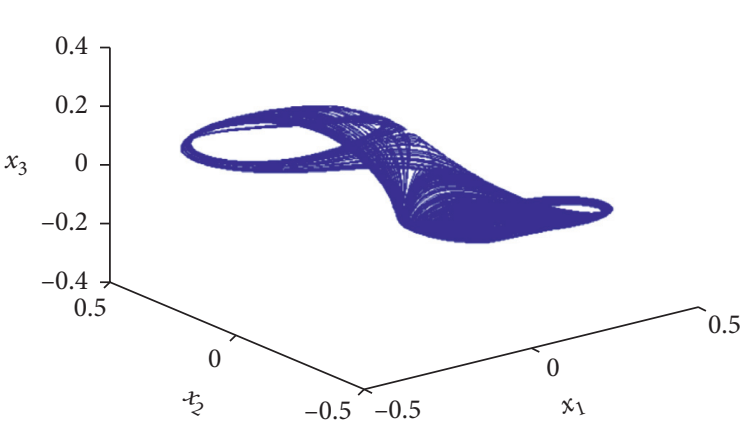

(e)

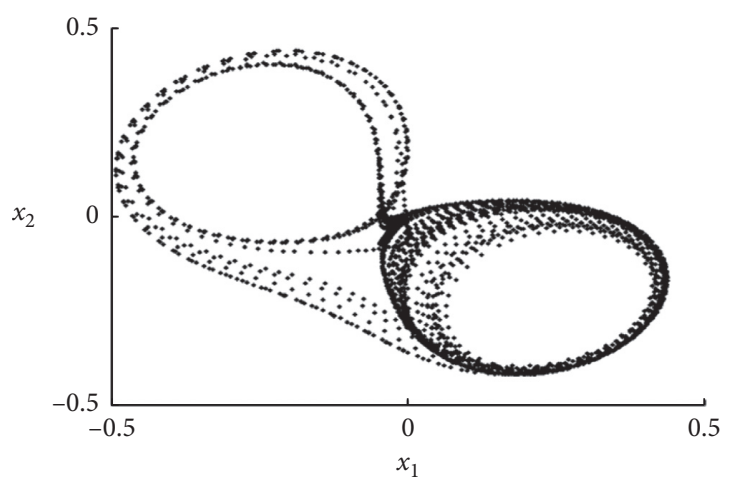

(f)

FIGURE 17: The chaotic oscillations of the cantilevered pipe conveying pulsating fluid are obtained when the external excitation is $F=6.5$. (a) Phase portrait on plane $\left(x_{1}, x_{2}\right)$, (b) waveform on plane $\left(t, x_{1}\right),(\mathrm{c})$ phase portrait on plane $\left(x_{3}, x_{4}\right)$, (d) waveform on plane $\left(t, x_{3}\right)$, (e) threedimensional phase portrait in space $\left(x_{1}, x_{2}, x_{3}\right)$, and (g) Poincare map on plane $\left(x_{1}, x_{2}\right)$.

irregular oscillations around the left-mode and the rightmode positions exist in the cantilevered pipe conveying pulsating fluid under the external harmonic excitation.

Moreover, the oscillations corresponding to different velocity parametric excitations and external excitations are consistent with the bifurcation diagrams of the cantilevered pipe conveying pulsating fluid under the external harmonic force, as shown in Figures 7 and 8. Based on the aforementioned analyses, it can be found that the flow rate and the external excitation can affect the nonlinear oscillations of the cantilevered pipe conveying pulsating fluid under the case of $1: 3$ internal resonance. When the velocity parametric excitation or the external excitation reaches a certain value, the multitime chaotic oscillations will happen in the cantilevered pipe conveying pulsating fluid, and the disorderly motions will lead to the destruction of the cantilevered pipe conveying pulsating fluid under the external harmonic force. Therefore, the velocity parametric excitation and the external excitation causing the multitime chaotic oscillations should be controlled when engineers design and use the infusion tube system.

\section{Conclusions}

The nonlinear resonant responses and multitime chaotic dynamics of the cantilevered pipe conveying pulsating fluid are investigated under the external harmonic force. Based on the nonlinear partial differential governing equation of motion for the cantilevered pipe conveying pulsating fluid derived by using Hamilton's principle, the 1:3 internal resonance and primary parametric resonance-1/2 subharmonic resonance are considered. A combination method of the method of multiple scales and Galerkin technique is utilized to obtain four-dimensional nonlinear averaged equations. Several amplitudefrequency response curves are obtained corresponding to the certain parameters. From the analysis of the amplitude-frequency response curves, it is found that there exist the hardening-spring type behaviors and the jumping phenomena. The jumping phenomena also occur in the amplitude-force response curves versus the flow velocity and external force.
Moreover, we find that the flow velocity, external force, and coupling degree of two order modes can affect the nonlinear vibrations of the cantilevered pipe conveying pulsating fluid under the external harmonic force. The nonlinear vibrations of the cantilevered pipe conveying pulsating fluid can be excited more easily with the increase of the flow velocity, external force, and coupling degree of two order modes. It is known that the nonlinear dynamic behaviors of the cantilevered pipe conveying pulsating fluid under the external harmonic force will be affected due to the flow rate and external excitation under the case of $1: 3$ internal resonance. It is observed that the multitime chaotic vibrations will occur for the cantilevered pipe conveying pulsating fluid when the velocity parametric excitation or external excitation reaches a certain value. From Figures 9-17, it is found that, for the first-order and thirdorder vibration modes, several-time nonlinear vibrations simultaneously or respectively happen around the rightmode and left-mode positions in the cantilevered pipe conveying pulsating fluid. Therefore, the velocity parametric excitation and external excitation should be controlled when engineers design and use the infusion tube systems.

\section{Data Availability}

All data generated or analyzed during this study are included in this published article.

\section{Conflicts of Interest}

The authors declare that there are no conflicts of interest regarding the publication of this paper.

\section{Acknowledgments}

The authors gratefully acknowledge the support of the National Natural Science Foundation of China (NNSFC) through Grant nos. 11672188, 11832002, and 11427801 and the Funding Project for Academic Human Resources Development in Institutions of Higher Learning under the Jurisdiction of Beijing Municipality (PHRIHLB). 


\section{References}

[1] D. M. Tang and E. H. Dowell, "Chaotic oscillations of a cantilevered pipe conveying fluid," Journal of Fluids and Structures, vol. 2, pp. 263-283, 1988.

[2] M. P. Païdoussis and F. C. Moon, "Nonlinear and chaotic fluid elastic vibrations of a flexible pipe conveying fluid," Journal of Fluids and Structures, vol. 2, pp. 567-591, 1988.

[3] C. Semler, G. X. Li, and M. P. Païdoussis, "The non-linear equations of motion of pipes conveying fluid," Journal of Sound and Vibration, vol. 5, pp. 577-599, 1994.

[4] M. P. Païdoussis, Fluid-Structure Interactions: Slender Structures and Axial Flow, Academic Press, London, UK, 2nd edition, 2014.

[5] P. J. Holmes, "Bifurcations to divergence and flutter in flowinduced oscillations: a finite dimensional analysis," Journal of Sound and Vibration, vol. 53, pp. 471-503, 1977.

[6] P. J. Holmes and J. Marsden, "Bifurcation to divergence and flutter in flow-induced oscillations: an infinite dimensional analysis," Automatica, vol. 14, pp. 367-384, 1978.

[7] Y. L. Huo and Z. M. Wang, "Dynamic analysis of a vertically deploying retracting cantilevered pipe conveying fluid," Journal of Sound and Vibration, vol. 360, pp. 224-238, 2016.

[8] M. Hosseini and R. Bahaadini, "Size dependent stability analysis of cantilever micro-pipes conveying fluid based on modified strain gradient theory," International Journal of Engineering Science, vol. 101, pp. 1-13, 2016.

[9] A. R. Askarian, H. Abtahi, R. D. Firouz-Abadi, H. Haddadpour, and E. H. Dowell, "Bending-torsional instability of a viscoelastic cantilevered pipe conveying pulsating fluid with an inclined terminal nozzle," Journal of Mechanical Science and Technology, vol. 32, pp. 2999-3008, 2018.

[10] E. Tubaldi, M. Amabili, and M. P. Païdoussis, "Fluid-structure interaction for nonlinear response of shells conveying pulsatile flow," Journal of Sound and Vibration, vol. 371, pp. 252-276, 2016.

[11] Y. C. Bai, W. D. Xie, X. F. Gao, and W. H. Xu, "Dynamic analysis of a cantilevered pipe conveying fluid with density variation," Journal of Fluids and Structures, vol. 81, pp. 638655, 2018.

[12] A. K. Bajaj, P. R. Sethna, and T. S. Lundgren, "Hopf bifurcation phenomena in tubes carrying a fluid," SIAM Journal on Applied Mathematics, vol. 39, pp. 213-230, 1980.

[13] N. Sri Namchchivaya, "Non-linear dynamics of supported pipe conveying pulsating fluid-I, subharmonic resonance," International Journal of Non-linear Mechanics, vol. 24, pp. 185-196, 1989.

[14] N. Sri Namchchivaya and W. M. Tien, "Non-linear dynamics of supported pipe conveying pulsating fluid-II, combination resonance," International Journal of Non-Linear Mechanics, vol. 24, pp. 197-208, 1989.

[15] A. Sarkar and M. P. Païdoussis, "A cantilever conveying fluid: coherent modes versus beam modes," International Journal of Non-Linear Mechanics, vol. 39, pp. 467-481, 2004.

[16] R. J. McDonald and N. Sri Namachchivaya, "Pipes conveying pulsating fluid near a 0:1 resonance: local bifurcations," Journal of Fluids and Structures, vol. 21, pp. 629-664, 2005.

[17] M. Yoshizawa, H. Nao, E. Hasegawa, and Y. Tsujioka, "Lateral vibration of a flexible pipe conveying fluid with pulsating flow," Transactions of the Japan Society of Mechanical Engineers, vol. 29, pp. 2243-2250, 2008.

[18] Y. Hou and G. H. Zeng, "Research on nonlinear dynamic characteristics of fluid-conveying pipes system," Advanced Materials Research, vol. 228-229, pp. 574-579, 2011.
[19] A. R. Setoodeh and S. Afrahim, "Nonlinear dynamic analysis of FG micro-pipes conveying fluid based on strain gradient theory," Composite Structures, vol. 116, pp. 128-135, 2014.

[20] M. Kheiri and M. P. Païdoussis, "On the use of generalized Hamilton's principle for the derivation of the equation of motion of a pipe conveying fluid," Journal of Fluids and Structures, vol. 50, pp. 18-24, 2014.

[21] C. B. Gan, S. Q. Guo, H. Lei, and S. X. Yang, "Random uncertainty modeling and vibration analysis of a straight pipe conveying fluid," Nonlinear Dynamics, vol. 77, pp. 503-519, 2014.

[22] T. Zhang, H. Ouyang, Y. O. Zhang, and B. L. Lv, "Nonlinear dynamics of straight fluid-conveying pipes with general boundary conditions and additional springs and masses," Applied Mathematical Modelling, vol. 40, pp. 7880-7900, 2016.

[23] Z. M. Wang and Y. Z. Liu, "Transverse vibration of pipe conveying fluid made of functionally graded materials using a symplectic method," Nuclear Engineering and Design, vol. 298, pp. 149-159, 2016.

[24] F. Liang, X.-D. Yang, Y.-J. Qian, and W. Zhang, "Free vibration analysis of pipes conveying fluid based on linear and nonlinear complex modes approach," International Journal of Applied Mechanics, vol. 9, no. 8, Article ID 1750112, 2017.

[25] F. Liang, X.-D. Yang, W. Zhang, and Y.-J. Qian, "Nonlinear free vibration of spinning viscoelastic pipes conveying fluid," International Journal of Applied Mechanics, vol. 10, no. 7, Article ID 1850076, 2018.

[26] F. Liang, X. D. Yang, Y. J. Qian, and W. Zhang, “Transverse free vibration and stability analysis of spinning pipes conveying fluid," International Journal of Mechanical Sciences, vol. 137, pp. 195-204, 2018.

[27] F. Liang, X. D. Yang, W. Zhang, and Y. J. Qian, "Dynamical modeling and free vibration analysis of spinning pipes conveying fluid with axial deployment," Journal of Sound Vibration, vol. 417, pp. 65-79, 2018.

[28] F. Liang, X. D. Yang, W. Zhang, and Y. J. Qian, "Coupled biflexural-torsional vibration of fluid-conveying pipes spinning about an eccentric axis," International Journal of Structural Stability and Dynamics, vol. 19, Article ID 1950003, 2019.

[29] F. Liang, X. D. Yang, W. Zhang, Y. J. Qian, and R. V. N. Melnik, "Parametric vibration analysis of pipes conveying fluid by nonlinear normal modes and a numerical iterative approach," Advances in Applied Mathematics and Mechanics, vol. 11, pp. 38-52, 2019.

[30] G. X. Li and M. P. Païdoussis, "Stability, double degeneracy and chaos in cantilevered pipes conveying fluid," International Journal of Non-Linear Mechanics, vol. 29, pp. 83-107, 1994.

[31] C. Semler and M. P. Païdoussis, "Nonlinear analysis of the parametric resonances of a planar fluid-conveying cantilevered pipe," Journal of Fluids and Structures, vol. 10, pp. 787-825, 1996.

[32] M. P. Païdoussis and C. Semler, "Non-linear dynamics of a fluid conveying cantilevered pipe with a small mass attached at the free end," International Journal of Non-Linear Mechanics, vol. 33, pp. 15-32, 1998.

[33] J. D. Jin and G. S. Zou, "Bifurcations and chaotic motions in the autonomous system of a restrained pipe conveying fluid," Journal of Sound and Vibration, vol. 260, pp. 783-805, 2003.

[34] M. H. Ghayesh, M. P. Païdoussis, and M. Amabili, "Nonlinear dynamics of cantilevered extensible pipes conveying fluid," Journal of Sound and Vibration, vol. 332, pp. 6405-6418, 2013. 
[35] L. Wang, Z. Y. Liu, A. Abdelkefi, Y. K. Wang, and H. L. Dai, "Nonlinear dynamics of cantilevered pipes conveying fluid: towards a further understanding of the effect of loose constraints," International Journal of Non-Linear Mechanics, vol. 95, 2017.

[36] A. R. Askarian, H. Haddadpour, R. D. Firouzabadi, and H. Abtahi, "Nonlinear dynamics of extensible viscoelastic cantilevered pipes conveying pulsatile flow with an end nozzle," International Journal of Non-linear Mechanics, vol. 91, pp. 22-35, 2017.

[37] T. Liu, W. Zhang, and J. F. Wang, "Nonlinear dynamics of composite laminated circular cylindrical shell clamped along a generatrix and with membranes at both ends," Nonlinear Dynamics, vol. 90, pp. 1393-1417, 2017.

[38] W. Zhang, T. Liu, A. Xi, and Y. N. Wang, "Resonant responses and chaotic dynamics of composite laminated circular cylindrical shell with membranes," Journal of Sound and Vibration, vol. 423, pp. 65-99, 2018.

[39] T. Liu, W. Zhang, J. J. Mao, and Y. Zheng, "Nonlinear breathing vibrations of eccentric rotating composite laminated circular cylindrical shell subjected to temperature, rotating speed and external excitations," Mechanical Systems and Signal Processing, vol. 127, pp. 463-498, 2019.

[40] W. Zhang, Y. Zheng, T. Liu, and X. Y. Guo, "Multi-pulse jumping double-parameter chaotic dynamics of eccentric rotating ring truss antenna under combined parametric and external excitations," Nonlinear Dynamics, vol. 98, pp. 761800, 2019.

[41] L. N. Panda and R. C. Kar, "Nonlinear dynamics of a pipe conveying pulsating fluid with parametric and internal resonances," Nonlinear Dynamics, vol. 49, pp. 9-30, 2007.

[42] Q. Ni, M. Tang, Y. Luo, Y. Wang, and L. Wang, "Internalexternal resonance of a curved pipe conveying fluid resting on a nonlinear elastic foundation," Nonlinear Dynamics, vol. 76, pp. 867-886, 2014.

[43] Y. L. Zhang and L. Q. Chen, "Steady-state response of pipes conveying pulsating fluid near a 2:1 internal resonance in the supercritical regime," International Journal of Applied Mechanics, vol. 6, Article ID 1450056, 2014.

[44] X. Y. Mao, H. Ding, and L. Q. Chen, "Steady-state response of a fluid-conveying pipe with $3: 1$ internal resonance in supercritical regime," Nonlinear Dynamics, vol. 86, pp. 795-809, 2016.

[45] Y. F. Zhang, M. H. Yao, W. Zhang, and B. C. Wen, "Dynamical modeling and multi-pulse chaotic dynamics of cantilevered pipe conveying pulsating fluid in parametric resonance," Aerospace Science and Technology, vol. 68, 2017.

[46] H. Ding, J. C. Ji, and L. Q. Chen, "Nonlinear vibration isolation for fluid-conveying pipes using quasi-zero stiffness characteristics," Mechanical Systems and Signal Processing, vol. 121, pp. 675-688, 2019.

[47] A. H. Nayfeh and D. T. Mook, Nonlinear Oscillations, Oxford University Press, Oxford, UK, 1979.

[48] J. E. Chen, W. Zhang, X. Y. Guo, and M. Sun, "Theoretical and experimental studies on nonlinear oscillations of symmetric cross-ply composite laminated plates," Nonlinear Dynamics, vol. 73, pp. 1697-1714, 2013.

[49] J. E. Chen, W. Zhang, J. Liu, and M. Sun, "Dynamic properties of truss core sandwich plate with tetrahedral core," Composite Structures, vol. 134, pp. 869-882, 2015.

[50] Z. Wei, Y. Li, B. Sang, Y. Liu, and W. Zhang, "Complex dynamical behaviors in a 3D simple chaotic flow with 3D stable or 3D unstable manifolds of a single equilibrium,"
International Journal of Bifurcation and Chaos, vol. 29, no. 7, Article ID 1950095, 2019.

[51] Y. J. Liu, F. Nazarimehr, A. J. M. Khalaf, A. Ahmed, and T. Hayat, "Detecting bifurcation points in a memristive neuron model," The European Physical Journal-Special Topics, vol. 228, pp. 1943-1950, 2019.

[52] Y. Liu, A. J. M. Khalaf, T. Hayat, A. Alsaedi, V.-T. Pham, and S. Jafari, "A complete investigation of the effect of external force on a 3D megastable oscillator," International Journal of Bifurcation and Chaos, vol. 30, no. 1, Article ID 2050012, 2020. 